\title{
Synopsis of the
}

Parasites of
Vertebrates
of Canada

\section{Edited by Murray J. Kennedy}

\section{Nematodes of Birds}

Pooi Leng Wong

Cheryl M. Bartlett

Lena N. Measures

Marilyn A. McNeill

Roy C. Anderson 
Other titles in this series

Synopsis of the Parasites of Vertebrates of Canada - Helminths and Protozoa of Terrestrial Mammals Murray J. Kennedy, 1986,90 p. $\$ 5.00$

Synopsis of the Parasites of Vertebrates of Canada - Ectoparasites of Terrestrial Mammals

Murray J. Kennedy and Robert A. Newman, 1986,109 p. $\$ 5.00$

Synopsis of the Digenea of Mammals of North America

Murray J. Kennedy, 1988, 83 p. $\$ 5.00$

Synopsis of the Parasites of Domesticated Mammals of Canada Murray J. Kennedy, 1988, 53 p. $\$ 5.00$

Synopsis of the Parasites of Vetebrates of Canada

- Parasites of Marine Mammals

Leo Margolis and Hisao P. Arai, 1989, 26 p. $\$ 5.00$

Synopsis of the Parasites of Vetebrates of Canada

- Ectoparasites of Birds

Terry A. Wheeler and William Threlfall, 1989,93 p. $\$ 5.00$

Synopsis of the Parasites of Vetebrates of Canada

- Haemoprotozoans of Birds

G. F. Bennett, C. B. Woodworth-Lynas and M. A. Bishop, 198947 p. $\$ 5.00$

Synopsis of the Parasites of Vertebrates of Canada

- Cestodes of Waterfowl

J. Daniel McLaughlin, 1989, 21 p. $\$ 5.00$

Copies of these publications are available from the Queen's Printer, 11510 Kingsway Avenue, Edmonton T5G 2Y2

Orders for these publications must be accompanied by a cheque that is payable to The Provincial Treasurer 


\title{
Synopsis of the
}

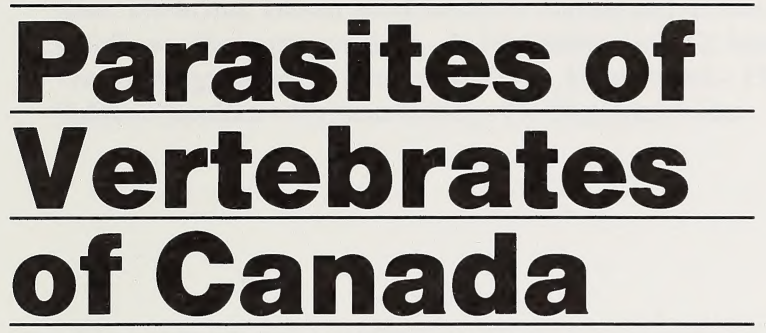

Edited by Murray J. Kennedy ${ }^{1}$

\section{Nematodes of Birds}

Pooi Leng Wong 2

Cheryl M. Bartlett ${ }^{3}$

Lena N. Measures ${ }^{4}$

Marilyn A. McNeill5

Roy C. Anderson? 2

\author{
1 Alberta Agriculture \\ Veterinary Laboratory \\ 6909 - 116 Street \\ Edmonton, Alberta \\ Canada T6H 4P2 \\ 2 Department of Zoology \\ College of Biological Science \\ University of Guelph \\ Guelph, Ontario \\ Canada N1G 2W1
}

${ }^{3}$ Department of Mathematics and Natural Sciences University College of Cape Breton

P.O. Box 5300

Sydney, Nova Scotia

Canada B1P 6L2

${ }^{4}$ Maurice Lamontagne Institute 850 , route de la Mer

P.O. Box 1000 Mont-Joli, Quebec

Canada G5H $3 Z 4$

5123 Foley Road Alymer, Quebec Canada J9H 2E6 



\section{ABSTRACT}

One hundred and sixty-two species of nematodes reported in birds in Canada are listed with their hosts, sites in hosts, synonyms, known distributions in Canada and references. The numbers of species according to superfamilies are as follows: Dioctophymatoidea - 2; Trichinelloidea - 12; Muspicioidea - 1; Rhabditoidea - 1; Strongyloidea - 4;

Trichostrongyloidea - 9; Heterakoidea - 5; Ascaridoidea - 15; Dracunculoidea - 2; Thelazioidea - 1; Spiruroidea - 1; Habronematoidea - 17; Acuarioidea - 45; Diplotriaenoidea - 15; Aproctoidea - 4 and Filarioidea - 26. 
Digitized by the Internet Archive in 2016 


\section{CONTENTS}

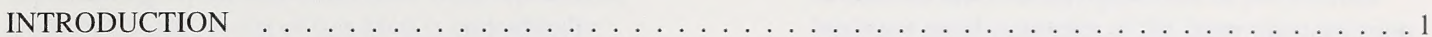

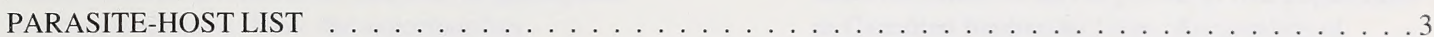

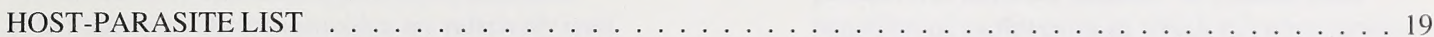

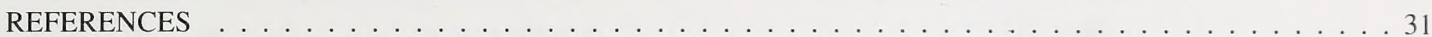

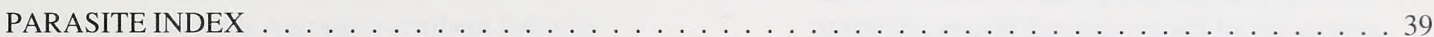

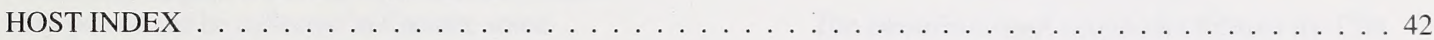



One hundred and sixty-two species of nematodes representing 16 superfamilies have been reported in birds in Canada. A list of this kind is undoubtedly biased because it reflects the interests of specialized workers in Canada. Thus, the superfamilies Acuarioidea, Ascaridoidea, Dioctophymatoidea, Filarioidea and Diplotriaenoidea are relatively well studied whereas other superfamilies are not well represented.

Most Canadian birds migrate to southern latitudes where they acquire a number of nematodes which may persist and be collected in Canada; some acuarioids are of this type. On the other hand, birds acquire some species on the breeding grounds in Canada; filarioids transmitted by biting flies and lice are good examples. Numerous species of nematodes acquired in Canada are short-lived and each spring and summer a new population of nematodes are acquired by the birds in Canada; acuarioids and dioctophymatoids of piscivorous birds are good examples of the latter phenomemon since the infective larvae persist in fish populations in Canadian freshwater lakes. Ascaridoids of passeriform birds are acquired in Canada from ingestion of earthworms in which infective larvae have persisted over winter.

Information in this synopsis excludes reports of microfilariae and diseases caused by nematodes.

The classification of nematodes follows the $\mathrm{CIH}$ Keys to the Nematode Parasites of Vertebrates by Anderson et al. (1974-1983). Names of birds are according to the American Ornithologists' Union Checklist of the Birds of North America (1983) and The Complete Checklist of Birds of the World by Howard and Moore (1984). 



\section{PARASITE-HOST LIST}

\section{SUBCLASS ADENOPHOREA ORDER ENOPLIDA}

\section{SUPERFAMILY DIOCTOPHYMATOIDEA FAMILY DIOCTOPHYMATIDAE SUBFAMILY EUSTRONGYLINAE}

Eustrongylides mergorum Rudolphi, 1809

Syns: Eustrongylides elegans (Olfers, 1816)

Spiroptera inflata Mehlis, 1846

Tropidocerca paradoxa Diesing, 1851

Site: stomach

Hosts: "mergansers" (1)

Uria aalge (2)

Uria lomvia (2)

Dist: BC, Nfld

Records: 1. Shillinger 1936 (BC); 2. Threlfall 1971 (Nfld)

Eustrongylides tubifex (Nitzsch in Rudolphi, 1819)

Syns: Strongylus tubifex

Eustrongylides perpapillatus Jagerskiold, 1909

Site: proventriculus

Hosts: Ardea herodias

Gavia immer

Mergus merganser

Mergus serrator

Dist: Ont, NB

Record: Measures 1987

\section{SUPERFAMILY TRICHINELLOIDEA FAMILY TRICHURIDAE SUBFAMILY CAPILLARIINAE}

Capillaria anatis (Schrank, 1790)

Site: caecum, small intestine, body cavity

Hosts: Anas rubripes $(1,4)$

Aythya collaris (3)

Bucephala clangula $(1,4)$

Lophodytes cucullatus (2)

Melanitta perspicillata (5)

Dist: BC, NB, Nfld, Ont, Que

Records: 1. Mawson 1956b (Que); 2.Bain and

Threlfall 1977 (Ont); 3. Noseworthy and Threlfall 1978 (Ont); 4. Mahoney and Threlfall 1978 (NB,

Nfld, Ont); 5. Bourgeois and Threlfall 1982 (BC,

$\mathrm{NB}, \mathrm{Nfld})$

Capillaria caudinflata (Molin, 1858)

Site: intestine

Host: Turdus migratorius

Dist: Que

Record: Mawson 1956b
Capillaria columbae (Rudolphi, 1819)

Site: duodenum, ileum

Host: Gallus gallus

Dist: Man

Record: Hodasi 1966

Capillaria contorta (Creplin, 1839)

Site: oesophagus

Hosts: Anas discors (1) Gallinago gallinago (2)

Larus argentatus (3)

Passerella iliaca (4)

Dist: Nfld, Que

Records: 1. Mawson 1956b (Que); 2. Threlfall 1968b; 3. Threlfall 1968c; 4. Jewer and Threlfall 1978

Capillaria falconis-nisi (Diesing, 1851)

Site: intestine

Hosts: "owl"

Dist: Que

Bubo virginianus

Record: Mawson 1956b

Capillaria mergi Madsen, 1945

Site: small intestine

Host: Bucephala clangula

Dist: Nfld, Ont

Record: Mahoney and Threlfall 1978

Capillaria nyrocinarum Madsen, 1945

Site: caecum, small intestine

Hosts: Melanitta fusca

Melanitta nigra

Melanitta perspicillata

Dist: BC, NB, Nfld

Record: Bourgeois and Threlfall 1982

Capillaria obsignata Madsen, 1945

Site: caecum

Hosts: Aechmophorus occidentalis $(2,3)$

Anas rubripes (1)

Podiceps auritus $(2,3)$

Podiceps grisegena $(2,3)$

Podiceps nigricollis $(2,3)$

Dist: Alta, NB, Nfld

Records: 1. Mahoney and Threlfall 1978 (NB, Nfld); 2. Stock and Holmes 1987 (Alta); 3. Stock and Holmes 1988 (Alta)

Capillaria ovopunctatum (Linstow, 1873)

Syn: Capillaria quiscala Read, 1949

Site: small intestine 
Hosts: Quiscalus quiscula versicolor $(1,2)$ Sturnus vulgaris (1)

Dist: Man, Que

Records: 1. Mawson 1956b (Que); 2. Hodasi 1963 (Man)

Capillaria spinulosa (Linstow, 1848)

Site: caecum

Host: Anas rubripes

Dist: NB, Nfld

Record: Mahoney and Threlfall 1978

Capillaria tridens (Dujardin, 1845)

Site: small intestine

Host: Pipilo erythropthalmus

Dist: Man

Record: Hodasi 1963

Capillaria species

Site: oesophagus

Hosts: "ducks, geese and swans" (2)

Anas platyrhynchos (domestic) (1)

Anas rubripes $(1,4,6)$

Bucephala clangula $(1,3)$

Ceryle alcyon (7)

Corvus brachyrhynchos (1)

Larus delawarensis (3)

Phasianus colchicus (5)

Dist: NB, Nfld, Ont, Que

Records: 1. Mawson 1956b (Que); 2. Karstad and Sileo 1971 (Ont); 3. Sileo et al. 1977 (Ont);

4. Mahoney and Threlfall 1978 (NB, Nfld, Ont);

5. Blanchard and Saint-Jacques 1979 (Que);

6. McLaughlin and Burt 1979 (NB); 7. Scott 1984 (Que)

\section{SUPERFAMILY MUSPICIOIDEA \\ FAMILY ROBERTDOLLFUSIDAE}

\section{Robertdollfusa species}

Site: oesophageal region of body cavity

Host: Larus argentatus

Dist: Nfld

Record: Threlfall 1968c

\section{SUBCLASS SECERNENTEA ORDER RHABDITIDA}

\section{SUPERFAMILY RHABDITOIDEA FAMILY STRONGYLOIDIDAE}

Strongyloides species

Site: intestine

Host: Ceryle alcyon

Dist: Que

Record: Scott 1984
ORDER STRONGYLIDA

\section{SUPERFAMILY STRONGYLOIDEA \\ FAMILY SYNGAMIDAE \\ SUBFAMILY SYNGAMINAE}

Cyathostoma bronchialis (Muehling, 1884)

Site: trachea

Host: Branta canadensis

Dist: Ont

Record: Fernando et al. 1973

Cyathostoma lari (Blanchard, 1849)

Site: trachea

Host: Larus argentatus

Dist: Nfld

Record: Threlfall 1968c

Cyathostoma species

Site: trachea

Host: "ducks, geese and swans"

Dist: Ont

Record: Karstad and Sileo 1971

Syngamus trachea Siebold, 1836

Site: trachea

Hosts: Agelaius phoenicus (2)

Ceryle alcyon (5)

Passerella iliaca (3)

Phasianus colchicus $(1,4)$

Seiurus noveboracensis (3)

Dist: Nfld, Ont, Que

Records: 1. Kingscote 1957 (Ont); 2. Rau et al. 1978 (Que); 3. Jewer and Threlfall 1978 (Nfld);

4. Blanchard and Saint-Jacques 1979 (Que);

5. Scott 1984 (Que)

\section{SUPERFAMILY TRICHOSTRONGYLOIDEA \\ FAMILY AMIDOSTOMATIDAE SUBFAMILY AMIDOSTOMATINAE}

Amidostomum acutum (Lundahl, 1848)

Site: gizzard, under lining

Hosts: Anas crecca (2)

Anas discors (2)

Anas rubripes (3)

Bucephala clangula (3)

Melanitta fusca (4)

Melanitta nigra (4)

Melanitta perspicillata (4)

Somateria mollissima (1)

Dist: BC, NB, Nfld, NS, Ont

Records: 1. Bishop and Threlfall 1974 (Nfld);

2. Turner and Threlfall 1975 (NB, NS); 3. Mahoney and Threlfall 1978 (NB, Nfld, Ont); 4. Bourgeois and Threlfall 1982 (BC, NB, Nfld) 
Site: gizzard, under lining

Hosts: "geese" $(1,6)$

"gosling" (5)

Anas acuta (9)

Anas platyrhynchos $(3,9)$

Branta canadensis $(4,10)$

Branta species (2)

Bucephala clangula (8)

Chen caerulescens (11)

Cygnus buccinator (8)

Cygnus columbianus (8)

Cygnus species (7)

Dist: BC, Man, Ont

Records: 1. Cram 1928 (Ont); 2. Swales 1933

(Ont); 3. Wickware 1940 (Ont); 4. Wickware

1941 (Ont); 5. McCraw 1952 (BC); 6. Oliver

1952 (Ont); 7. Mawson 1956c (unspecified);

8. MacNeill 1970 (BC); 9. Crichton and Welch

1972 (Man); 10. MacNeill and Barnard 1978

(BC); 11. Tuggle and Crites 1984 (Ont)

Amidostomum cygni Wehr, 1933

Site: gizzard, under lining

Hosts: Cygnus columbianus

Gavia stellata

Dist: Que

Somateria spectabilis

Record: Mawson 1956c

Amidostomum spatulatum Baylis, 1932

Site: gizzard, under lining

Hosts: Chen caerulescens (2)

Chen caerulescens atlanticus (1)

Dist: Ont

Records: 1. Mawson 1956c (unspecified); 2.

Tuggle and Crites 1984 (Ont)

\section{Amidostomum species}

Site: gizzard, under lining

Hosts: "ducks, geese and swans" (3)

"wild swans" (4)

Anas clypeata (2)

Anas discors (1)

Chen caerulescens (4)

Cygnus columbianus (5)

Cygnus buccinator (5)

Melanitta perspicillata (6)

Dist: BC, Man, NB, Nfld, Ont

Records: 1. Buscher 1965 (Man); 2. Buscher

1966 (Man); 3. Karstad and Sileo 1971 (Ont);

4. MacNeill and Barnard 1978 (BC); 5. McKelvey

and MacNeill 1981 (BC); 6. Bourgeois and

Threlfall 1982 (BC, NB, Nfld)
Epomidiostomum crami Wetzel, 1931

Site: gizzard, under lining

Hosts: Chen caerulescens (3)

Aythya collaris (2)

Bucephala clangula (1)

Dist: NB, Ont

Records: 1. Mahoney and Threlfall 1978 (Ont);

2. Noseworthy and Threlfall 1978 (NB, Ont);

3. Tuggle and Crites 1984 (Ont)

Epomidiostomum uncinatum (Lundahl, 1848)

Site: gizzard, under lining

Hosts: Anas acuta $(1,3)$

Anas clypeata (1)

Anas crecca (4)

Anas discors $(2,4)$

Anas platyrhynchos (3)

Anas rubripes (5)

Anas strepera (1)

Melanitta nigra (6)

Dist: BC, Man, NB, Nfld, NS

Records: 1. Buscher 1965 (Man); 2. Buscher

1966 (Man); 3. Crichton and Welch 1972 (Man);

4. Turner and Threlfall 1975 (NB, NS); 5. Mahoney

and Threlfall 1978 (NB, Nfld); 6. Bourgeois and

Threlfall 1982 (BC, NB, Nfld)

\section{FAMILY TRICHOSTRONGYLIDAE SUBFAMILY TRICHOSTRONGYLINAE}

Trichostrongylus tenuis (Mehlis, 1846)

Site: caecum

Hosts: Anser anser (1)

Chen caerulescens atlanticus (2)

Chen caerulescens (2)

Dist: NWT, Ont

Records: 1. Griffiths 1947 (Ont); 2. Mawson 1956c (NWT)

\section{FAMILY ORNITHOSTRONGYLIDAE SUBFAMILY ORNITHOSTRONGYLINAE}

Ornithostrongylus quadriradiatus (Stevenson, 1904)

Site: unspecified

Hosts: Columba livia (1)

Columba species (2)

Dist: Ont, unspecified

Records: 1. Glover 1947 (Ont); 2. Speckman 1976 (unspecified) 


\section{ORDER ASCARIDIDA}

\section{SUPERFAMILY HETERAKOIDEA \\ FAMILY HETERAKIDAE \\ SUBFAMILY HETERAKINAE}

Heterakis gallinarum (Schrank, 1788)

Syn: Heterakis gallinae (Gmelin, 1790)

Site: caecum

Host: Gallus gallus

Dist: Man, Sask

Records: 1. Hodasi 1966 (Man); 2. Riddell and

Gajadhar 1988 (Sask)

Heterakis isolonche Linstow, 1906

Site: caecum

Host: Phasianus colchicus

Dist: Ont

Records: 1. Swales 1933; 2. Mitchell 1953

\section{FAMILY ASCARIDIIDAE}

Ascaridia compar (Schrank, 1790)

Site: caecum

Host: Lagopus lagopus

Dist: Nfld

Record: Threlfall 1968b

Ascaridia galli (Schrank, 1788)

Syns: Ascaridia lineata Schneider, 1866

Ascaridia bonasae Wehr, 1940

Site: caecum

Hosts: Bonasa umbellus $(1,3,4)$

Gallus gallus (2)

Dist: Man, Ont

Records: 1. Clarke 1936 (Ont); 2. Hodasi 1966

(Man); 3. Anon. 1967 (Ont): 4. Fyvie and

Addison 1979 (Ont)

Ascaridia species fourth-stage larvae

Site: intestine

Host: Meleagris gallopavo

Dist: Ont

Record: Hemsley 1971

\section{SUPERFAMILY ASCARIDOIDEA \\ FAMILY ANISAKIDAE \\ SUBFAMILY GOEZIINAE}

Contracaecum anasi Mawson, 1956

Site: unspecified

Host: Anas rubripes

Dist: Que

Record: Mawson 1956a
Contracaecum magnicollare Johnston and

Mawson, 1941

Site: unspecified

Host: Diomedea species

Dist: BC

Record: Mawson 1956a

Contracaecum microcephalum (Rudolphi, 1809)

Site: gizzard, under lining

Host: Botaurus lentiginosus

Dist: Nfld

Record: Threlfall 1968b

Contracaecum ovale Linstow, 1907

Site: intestine

Hosts: Aechmophorus occidentalis

Podiceps auritus

Podiceps grisegena

Podiceps nigricollis

Dist: Alta

Records: 1. Stock and Holmes 1987; 2. Stock and Holmes 1988

Contracaecum pelagicum Johnston and Mawson, 1942

Site: unspecified

Host: Diomedea species

Dist: BC

Record: Mawson 1956a

Contracaecum podicipitis Johnston and Mawson, 1949

Site: unspecified

Host: Podilymbus podiceps

Dist: unspecified

Record: Mawson 1956a

Contracaecum spiculigerum (Rudolphi, 1809)

Site: gizzard

Hosts: Alca torda (5)

Larus argentatus (4)

Larus marinus (2)

Phalacrocorax auritus (1)

Podilymbus podiceps (3)

Rissa tridactyla (2)

Uria aalge (5)

Uria lomvia (5)

Dist: NB, Nfld

Records: 1. Mawson 1956a (NB); 2. Threlfall 1968a (Nfld); 3. Threlfall 1968b (Nfld); 4. Threlfall 1968c (Nfld); 5. Threlfall 1971 (Nfld)

Contracaecum yamaguti Mawson, 1956

Site: unspecified

Hosts: Mergus merganser

Dist: unspecified

Record: Mawson 1956a 


\section{Contracaecum species}

Site: proventriculus (1) unspecified (2)

Hosts: Larus argentatus (2)

Puffinus gravis (1)

Dist: Nfld

Records: 1. Bourgeois and Threlfall 1979

(Nfld); 2. Threlfall 1968c (Nfld)

\section{FAMILY ASCARIDIDAE SUBFAMILY TOXOCARINAE}

Porrocaecum brevispiculum Webster, 1943

Site: small intestine

Host: Passerella iliaca

Dist: Nfld

Records: 1. Threlfall 1968b 2. Jewer and Threlfall 1978

Porrocaecum depressum (Zeder, 1800)

Site: intestine

Host: Bubo virginianus

Dist: Alta, Man

Records: 1. Morgan and Schiller 1950 (Man);

2. Ramalingam and Samuel 1978 (Alta)

Porrocaecum ensicaudatum (Zeder, 1800)

Site: gizzard, under lining and small intestine

Hosts: Corvus brachyrhynchos (1)

Molothrus ater (1)

Quiscalus quiscula $(1,2)$

Sturnus vulgaris (2)

Turdus migratorius $(1,2)$

Dist: Ont, Que

Records: 1. Mawson 1956a (Que); 2. McNeill 1988 (Ont)

Porrocaecum semiteres (Zeder, 1800)

Site: unspecified

Host: Larus argentatus

Dist: Nfld

Record: Threlfall 1968c

Porrocaecum trichuriforme Mawson, 1956

Site: unspecified

Hosts: Bubo virginianus

Dist: Ont

Falco peregrinus anatum

Record: Mawson 1956a

Porrocaecum species

Site: intestine (2), unspecified (1)

Hosts: Agelaius phoeniceus (2)

Corvus brachyrhynchos (1)

Turdus migratorius (1)

Dist: Que

Records: 1. Rayner 1932; 2. Rau et al. 1978
Ascarididae, unidentified larvae

Site: intestine

Hosts: Corvus brachyrhynchos (1)

Meleagris gallopavo (2)

Dist: Man, Nfld

Records: 1. Andrews and Threlfall 1975

(Nfld); 2. Neufeld 1987 (Man)

\section{ORDER SPIRURIDA}

\section{SUPERFAMILY DRACUNCULOIDEA FAMILY DRACUNCULIDAE}

Avioserpens nana Mawson, 1957

Site: unspecified

Host: Ardea herodias

Dist: Que

Record: Mawson 1957

Avioserpens species

Site: connective tissue between oesophagus and trachea

Hosts: Botaurus lentiginosus Ixobrychus exilis

Dist: Ont, Que

Record: Gibson 1973

\section{SUPERFAMILY THELAZIOIDEA FAMILY THELAZIDAE SUBFAMILY OXYSPIRURINAE}

Oxyspirura petrowi Skrjabin, 1929

Syn: Oxyspirura lumsdeni Addison and Anderson, 1969

Site: orbits

Hosts: Bonasa umbellus

Centrocercus urophasianus

Tympanuchus phasianellus

Tympanuchus cupido

Dist: Ont, Sask

Record: Addison and Anderson 1969

\section{SUPERFAMILY SPIRUROIDEA FAMILY SPIROCERCIDAE SUBFAMILY ASCAROPSINAE}

Physocephalus sexalatus third-stage larvae

Site: serosa of small and large intestine and mesentery

Hosts: Bartramia longicauda Numenius americanus

Dist: Alta, Man

Record: Bartlett et al. 1987 


\section{SUPERFAMILY HABRONEMATOIDEA \\ FAMILY HABRONEMATIDAE \\ SUBFAMILY HABRONEMATINAE}

Cyrnea americanum Chandler, 1941

Site: proventriculus, gizzard

Host: Bubo virginianus

Dist: Alta

Record: Ramalingam and Samuel 1978

Cyrnea longispicula Walton, 1927

Site: proventriculus, gizzard

Host: Nyctea scandiaca

Dist: Alta

Record: Ramalingam and Samuel 1978

Habronema mansioni Seurat, 1914

Syns: Spiroptera longistriata Molin, 1859

Cheilospirura longistriata

Site: gizzard, under lining

Hosts: Buteo jamaicensis borealis

Dist: Que, Sask

Falco peregrinus anatum

Record: Mawson 1956e

\section{SUBFAMILY HISTIOCEPHALINAE}

Stellocaronema skrjabini Gilbert, 1930

Syn: Stellocaronema glareolae Mawson, 1968

Site: gizzard, under lining

Hosts: Bartramia longicauda (3)

Calidris alba $(1,2,3)$

Calidris alpina $(1,2,3)$

Calidris himantopus (3)

Calidris mauri (1)

Calidris minutilla (3)

Calidris pusilla (3)

Catoptrophorus semipalmatus inornatus $(2,3)$

Charadrius semipalmatus (2)

Limosa fedoa $(2,3)$

Limnodromus scolopaceus (3)

Phalaropus lobatus (2)

Tringa melanoleuca (2)

Dist: Alta, BC, Man, NB, Sask

Records: 1. Adams and Gibson 1969 (BC);

2. Wong 1985 (Alta, Man); 3. Wong and

Anderson in press (Alta, NB, Sask)

\section{FAMILY TETRAMERIDAE SUBFAMILY TETRAMERINAE}

Microtetrameres bubo Schell, 1953

Site: proventriculus, gizzard

Host: Bubo virginianus

Dist: Alta

Record: Ramalingam and Samuel 1978
Microtetrameres canadensis Mawson, 1956

Site: proventriculus

Hosts: Ardea herodias

Dist: Que

Nyctea scandiaca

Record: Mawson 1956d

Microtetrameres helix Cram, 1927

Site: proventriculus

Host: Corvus brachyrhynchos

Dist: Man, Que

Records: 1. Mawson 1956e (Que); 2. Hodasi 1963 (Man)

Microtetrameres species

Site: proventriculus

Hosts: Agelaius phoeniceus $(2,5)$

Nyctea scandiaca (3)

Quiscalus quiscula versicolor (1)

Tyrannus tyrannus (4)

Dist: Alta, Man, Que

Records: 1. Hodasi 1963 (Man); 2. Rau et al. 1978 (Que); 3. Ramalingam and Samuel 1978 (Alta); 4. MacKenzie et al. 1979 (Man); 5. Hood and Welch 1980 (Man)

Tetrameres ardamericanus Boyd, 1966

Site: proventriculus

Host: Ardea herodias

Dist: Ont

Record: Boyd 1966

Tetrameres crami Swales, 1933

Site: proventriculus

Host: Anas platyrhynchos (domestic)

Dist: Ont, Que

Records: 1. Swales 1933a,b (Ont); 2. Swales 1936 (Que)

Tetrameres fissispina (Diesing, 1861)

Syns: Tropidocerca fissispina

Tropisurus fissispina

Acanthophorus horridus Linstow, 1876

Acanthophorus tenuis Linstow, 1876

Filaria pulicis Linstow, 1894

Site: proventriculus

Hosts: Anas rubripes (1)

Aythya collaris (2)

Melanitta fusca (3)

Melanitta nigra (3)

Melanitta perspicillata (3)

Dist: BC, NB, Nfld, Ont

Records: 1. Mahoney and Threlfall 1978 (NB,

Nfld); 2. Noseworthy and Threlfall 1978 (Ont); 3.

Bourgeois and Threlfall 1982 (BC, NB, Nfld) 
Site: proventriculus

Host: Phoenicopterus ruber

Dist: Nfld

Record: Threlfall 1981

Tetrameres ryjikovi Khuan, 1961

Site: proventriculus

Hosts: Anas crecca (1)

Anas discors (1)

Bucephala clangula (2)

Dist: Nfld, Ont

Records: 1. Turner and Threlfall 1975 (Nfld);

2. Mahoney and Threlfall 1978 (Nfld, Ont)

Tetrameres somateriae Ryzhikov, 1963

Site: proventriculus

Hosts: Melanitta fusca (1)

Melanitta nigra (1)

Melanitta perspicillata (1)

Somateria mollissima (2)

Dist: BC, NB, Nfld

Records: 1. Bourgeois and Threlfall 1982 (BC,

NB, Nfld); 2. Bishop and Threlfall 1974 (Nfld)

Tetrameres spinosa (Maplestone, 1931)

Syn: Echinuria spinosa

Site: proventriculus

Hosts: Melanitta fusca

Melanitta nigra

Melanitta perspicillata

Dist: BC, NB, Nfld

Record: Bourgeois and Threlfall 1982

\section{Tetrameres species}

Site: proventriculus

Hosts: "ducks, geese and swans" (1)

Agelaius phoeniceus (7)

Anas rubripes (4)

Aythya collaris (5)

Bucephala clangula (4)

Catoptrophorus semipalmatus inornatus (9)

Larus delawarensis (2)

Melanitta fusca (8)

Melanitta perspicillata (8)

Lophodytes cucullatus (3)

Puffinus gravis (6)

Dist: Alta, BC, Man, NB, Nfld, Ont

Records: 1. Karstad and Sileo 1971 (Ont);

2. Sileo et al. 1977 (Ont); 3. Bain and Threlfall

1977 (Ont); 4. Mahoney and Threlfall 1978

(unspecified); 5. Noseworthy and Threlfall 1978

(Ont); 6. Bourgeois and Threlfall 1979 (BC, NB,

Nfld); 7. Hood and Welch 1980 (Man); 8.

Bourgeois and Threlfall 1982 (NB, Nfld, Ont); 9.

Wong and Anderson unpublished data (Alta)
Geopetitia aspiculata Webster, 1971

Site: proventriculus

Hosts: Anitibyx armatus

Chloropsis hardwickei

Irena puella

Minla cyanouroptera

Myiophoneus caeruleus

Phoeniculus purpureus

Pitangus sulphuratus

Pycnonotus cafer

Pycnonotus jocosus

Saltator coerulescens

Tachyphonus rufus

Thraupis episcopus

Thraupis palmarum

Dist: Man (all the birds were from the

Assiniboine Park Zoo, Winnipeg)

Record: Bartlett et al. 1984

\section{SUPERFAMILY ACUARIOIDEA \\ FAMILY ACUARIIDAE \\ SUBFAMILY ACUARIINAE}

Acuaria anthuris (Rudolphi, 1819)

Syns: Spiroptera anthuris

Filaria anthuris

Dispharagus anthuris

Acuaria ornata (Gendre, 1912)

Site: gizzard, under lining

Host: Corvus brachyrhynchos

Dist: Ont, Que

Records: 1. Mawson 1956e (Que); 2. Wong unpublished data (Ont)

Acuaria species

Site: gizzard, under lining

Hosts: "ducks, geese and swans" (1)

Numenius americanus (3)

Tyrannus tyrannus (2)

Tyrannus verticalis (2)

Dist: Alta, Man, Ont

Records: 1. Karstad and Sileo 1971 (Ont);

2. MacKenzie et al. 1979 (Man); 3. Wong and Anderson unpublished data (Alta)

Cheilospirura spinosa (Cram, 1927)

Syn: Acuaria spinosa

Site: gizzard, under lining

Hosts: Bonasa umbellus (1)

Callipepla californica (2)

Tympanuchus phasianellus (1)

Dist: BC, Ont

Records: 1. Clarke 1936 (Ont); 2. Chandler 1970 (BC) 
Chevreuxia americana Schmidt, 1968

Site: gizzard, under lining

Hosts: Charadrius vociferus

Pluvialis squatarola

Recurvirostra americana

Dist: Alta, Man

Record: Wong and Anderson unpublished data

Cosmocephalus capellae Yamaguti, 1935

Site: oesophagus

Host: Gallinago gallinago

Dist: Ont

Record: Threlfall 1968d

Cosmocephalus obvelatus (Creplin, 1825)

Syns: Spiroptera obvelatus

Filaria obvelatus

Dispharagus obvelatus

Cosmocephalus diesingi Molin, 1858

Spiroptera adunca Stossich, 1892

Cosmocephalus aduncus Yorke and

Maplestone, 1926

Cosmocephalus obvelatus magnus

Vasilkova, 1926

Cosmocephalus faridi Khalil, 1931

Cosmocephalus firlottei Rao, 1951

Cosmocephalus tanakai Rodrigues and

Vicente, 1963

Site: oesophagus

Hosts: Bombycilla cedrorum (3)

Catoptrophorus semipalmatus inornatus (8)

Larus argentatus $(1,4)$

Larus delawarensis $(6,7)$

Larus marinus (2)

Limosa fedoa (8)

Phalaropus tricolor (8)

Rissa tridactyla (2)

Tringa flavipes (8)

Uria aalge (5)

Dist: Alta, Nfld, Ont, Que

Records: 1. Rao 1951 (Que); 2. Threlfall 1968a

(Nfld); 3. Threlfall 1968b (Nfld) 4. Threlfall

1968c (Nfld); 5. Threlfall 1971 (Nfld); 6. Sileo

et al. 1977 (Ont); 7. Anderson and Wong 1981

(Ont); 8. Wong and Anderson unpublished data

(Alta)

Desportesius triaenucha (Wright, 1879)

Syns: Filaria triaenucha

Acuaria triaenucha

Streptocara triaenucha

Desportesius canadensis Mawson, 1956

Site: gizzard, under lining

Hosts: Ardea herodias (2)

Botaurus lentiginosus $(1,2)$

Dist: Ont, Que
Records: 1. Wright 1879 (Ont); 2. Mawson 1956d (Que)

Dispharynx nasuta (Rudolphi, 1819)

Syns: Spiroptera nasuta

Dispharagus nasutus

Acuaria (Dispharynx) nasuta

Cheilospirura nasuta

Dispharagus spiralis Molin, 1858

Acuaria spiralis

Dispharagus nasutus Piana, 1897

Dispharagus columbae Bridre, 1910

Site: proventriculus

Hosts: Agelaius phoeniceus $(1,2)$

Charadrius vociferus (3)

Dist: Alta, Man, Que

Records: 1. Rau et al. 1978 (Que); 2. Hood and Welch 1980 (Man); 3. Wong and Anderson unpublished data (Alta)

Echinuria borealis Mawson, 1956

Site: proventriculus

Hosts: Clangula hyemalis (1)

Melanitta perspicillata (3)

Somateria mollissima (2)

Somateria spectabilis (1)

Dist: BC, NB, Nfld, Que

Records: 1. Mawson 1956d (Que); 2. Bishop

and Threlfall 1974 (Nfld); 3. Bourgeois and

Threlfall 1982 (BC, NB, Nfld)

Echinuria hypognatha Wehr, 1937

Site: proventriculus

Hosts: Melanitta nigra

Melanitta perspicillata

Dist: BC, NB, Nfld

Record: Bourgeois and Threlfall 1982

Echinuria parva Cram, 1928

Site: proventriculus

Hosts: Branta canadensis $(1,3)$

Branta species (2)

Dist: Ont

Records: 1. Cram 1928; 2. Swales 1933;

3. Wickware 1941

Echinuria uncinata (Rudolphi, 1819)

Syns: Spiroptera uncinata

Dispharagus uncinata

Acuaria (Hamannia) uncinata

Hamannia uncinata

Echinuria jugadornata Soloviev, 1912

Site: proventriculus

Hosts: Anas acuta $(2,4)$

Anas discors $(2,3)$

Anas platyrhynchos $(1,2,4)$

Aythya americana (2) 
Aythya valisineria (2)

Bucephala albeola (2)

Calidris alba (6)

Calidris bairdii (6)

Calidris pusilla (6)

Catoptrophorus semipalmatus inornatus (6)

Cygnus buccinator (2)

Limosa fedoa (6)

Phalaropus tricolor (6)

Somateria mollissima (5)

Dist: Alta, Man, NB, Nfld, Sask

Records: 1. Mawson 1956e (Alta); 2. Cornwell 1963 (Man); 3. Buscher 1966 (Man); 4. Crichton and Welch 1972 (Man); 5. Bishop and Threlfall 1974 (Nfld); 6. Wong and Anderson unpublished data (Alta, Man, NB, Sask)

Paracuaria adunca (Creplin, 1846)

Syns: Spiroptera adunca

Dispharagus aduncus

Filaria tridentata Linstow, 1877

Spiroptera tridentata

Paracuaria tridentata

Rusguniella transcaucasica Solonitsin, 1932

Streptocara transcaucasica

Paracuaria macdonaldi Rao, 1951

Streptocara rissae Kreis, 1958

Site: gizzard, under lining

Hosts: Ardea herodias (2)

Cepphus columba (2)

Diomedea nigripes (2)

Gavia species (2)

Larus argentatus $(1,2,6)$

Larus canus (2)

Larus delawarensis $(2,6)$

Larus glaucescens (2)

Larus marinus (2)

Larus pipixcan (2)

Larus thayeri (2)

Melanitta perspicillata (2)

Podiceps auritus (2)

Rissa tridactyla (3)

Somateria mollissima (6)

Uria aalge (2)

Dist: Atlantic coast, BC, midwest, Nfld, Ont

Records: 1. Rao 1951 (Que); 2. Gibson 1968

(Atlantic coast, BC, midwest, Ont); 3. Threlfall 1968a (Nfld); 4. Threlfall 1968c (Nfld); 5. Bishop and Threlfall 1974 (Nfld); 6. Wong and Anderson 1982a (Ont)

Pectinospirura argentata Wehr, 1933

Syn: Pectinospirura sobolevi Turemuratov, 1965

Site: proventriculus

Hosts: Larus delawarensis (1)

Numenius americanus (2)
Dist: Alta, Ont

Records: 1. Wong and Anderson 1982b (Ont);

2. Wong and Anderson unpublished data (Alta)

Skrjabinocerca prima Shikhobalova, 1930

Site: oesophagus

Hosts: Catoptrophorus semipalmatus inornatus

Charadrius vociferus (2)

Phalaropus tricolor (2)

Pluvialis squatarola (2)

Recurvirostra americana $(1,2)$

Dist: Alta

Records: 1. Wong et al. 1987; 2. Bartlett et al. 1989

Skrjabinoclava bakeri Wong and Anderson, 1988 for (1987)

Site: proventriculus

Host: Calidris pusilla

Dist: Alta, NB, NS, Ont

Record: Wong and Anderson in press

Skrjabinoclava bartlettae Wong and Anderson, 1988 for (1987)

Site: proventriculus

Host: Pluvialis squatarola

Dist: Alta

Record: Wong and Anderson in press

Skrjabinoclava inornatae Wong and Anderson, 1988 for (1987)

Syn: Skrjabinoclava horrida Wong and Anderson, 1983

Site: proventriculus

Hosts: Catoptrophorus semipalmatus inornatus (2)

Larus delawarensis (1)

Dist: Alta, Man, Ont

Records: 1. Wong and Anderson 1983 (Ont); 2. Wong and Anderson in press (Alta, Man)

Skrjabinoclava kritscheri Wong and Anderson, 1988 for (1987)

Site: proventriculus

Host: Limosa fedoa

Dist: Alta, Man

Record: Wong and Anderson in press

Skrjabinoclava morrisoni Wong and Anderson, 1988 for (1987)

Site: proventriculus

Host: Calidris pusilla

Dist: Alta, Man, NB, NS, Ont, Sask

Record: Wong and Anderson in press 
Skrjabinoclava pusillae Wong and Anderson, 1988

for (1987)

Site: proventriculus

Hosts: Calidris pusilla

Charadrius semipalmatus

Dist: Alta, Man, NB, NS, Ont, Sask

Record: Wong and Anderson in press

Skrjabinoclava tupacincai Wong and Anderson, 1988 for (1987)

Site: proventriculus

Hosts: Calidris alba

Calidris alpina

Calidris pusilla

Dist: Man, NB, Sask

Record: Wong and Anderson in press

Unidentified Skrjabinoclava larvae

Site: proventriculus

Hosts: Calidris alba

Calidris pusilla

Charadrius semipalmatus

Dist: Alta, Man, NB, NS, Ont, Sask

Record: Wong and Anderson in press

Syncuaria squamata (Linstow, 1883)

Syns: Filaria squamata

Dispharagus squamatus

Acuaria squamata

Hamannia squamata

Echinuria squamata

Skrjabinocara squamata

Chordocephalus squamata

Skrjabinocara schikhobalovi Guschanskaya,

1950

Skrjabinocara skrjabini Guschanskaya, 1950

Skrjabinocara tiofejevi Guschanskaya, 1950

Skrjabinocara viktori Guschanskaya, 1950

Site: gizzard, under lining

Host: Phalacrocorax auritus

Dist: Ont

Record: Wong et al. 1986

Synhimantus species

Site: gizzard, under lining

Host: Botaurus lentiginosus

Dist: Nfld

Record: Threlfall 1968b

\section{SUBFAMILY SEURATINAE}

Aviculariella alcyona Wehr, 1931

Site: gizzard, under lining

Host: Ceryle alcyon

Dist: Ont, Que
Records: 1. Mawson 1956e (Que); 2. Boyd and

Fry 1971 (Ont); 3. Anderson et al. 1980 (Ont); 4. Scott (Que)

Molinacuaria bendelli (Adams and Gibson, 1969)

Syn: Ancyracanthopsis bendelli

Site: gizzard, under lining

Host: Dendragapus obscurus fuliginosus

Dist: BC

Record: Adams and Gibson 1969

Seuratia shipleyi (Stossich, 1900)

Syns: Gnathostoma shipleyi

Rictularia shipleyi

Prionostemma shipleyi

Rictularia paradoxa Linstow, 1903

Acuaria pelagica Seurat, 1916

Prionostemma pelagicum

Site: gizzard, under lining

Host: Puffinus gravis

Dist: Nfld

Record: Bourgeois and Threlfall 1979

Stegophorus stellae-pollaris (Parona, 1901)

Syns: Histiocephalus stellae-pollaris

Streptocara stellae-pollaris

Yseria stellae-pollaris

Site: gizzard, under lining

Host: Uria lomvia

Dist: Nfld

Record: Threlfall 1971

Stegophorus species

Site: gizzard, under lining

Host: Puffinus gravis

Dist: Nfld

Record: Bourgeois and Threlfall 1979

Streptocara californica (Gedoelst, 1919)

Syns: Yseria californica

Skrjabinobronema californica

Site: gizzard, under lining

Hosts: Anas platyrhynchos (1)

Aythya marila (1)

Aythya valisineria (1)

Bucephala islandica (1)

Clangula hyemalis (1)

Melanitta fusca $(1,2)$

Melanitta nigra $(1,2)$

Melanitta perspicillata $(1,2)$

Mergus merganser (1)

Mergus serrator (1)

Dist: BC, NB, Nfld

Records: 1. Gibson 1968 (BC); 2. Bourgeois and Threlfall 1982 (BC, NB, Nfld) 
Streptocara crassicauda (Creplin, 1829)

Syns: Spiroptera crassicauda

Dispharagus crassicauda

Streptocara crassicauda anseri Skrjabin, 1916

Site: gizzard, under lining

Hosts: Anas acuta (1)

Anas discors (2)

Anas platyrhynchos (1)

Melanitta fusca (4)

Melanitta nigra (4)

Melanitta perspicillata (4)

Lophodytes cucullatus (3)

Dist: BC, Man, NB, Nfld, Ont

Records: 1. Crichton and Welch 1972 (Man);

2. Turner and Threlfall 1975 (Nfld); 3. Bain and

Threlfall 1977 (Ont); 4. Bourgeois and Threlfall

1982 (BC, NB, Nfld)

Streptocara c. crassicauda Gibson, 1968

Site: gizzard, under lining

Hosts: Actitis macularia

Anas clypeata

Anas platyrhynchos

Aythya marila

Aythya valisineria

Bucephala clangula

Bucephala islandica

Cepphus columba

Clangula hyemalis

Larus thayeri

Lophodytes cucullatus

Melanitta nigra

Melanitta perspicillata

Mergus merganser

Mergus serrator

Oxyura jamaicensis

Dist: BC

Tympanuchus phasianellus

Record: Gibson 1968

Streptocara formosensis Sugimoto, 1930

Site: gizzard, under lining

Hosts: Anas platyrhynchos (1)

Aythya collaris (4)

Bucephala albeola (1)

Bucephala clangula $(1,3)$

Bucephala islandica (1)

Clangula hyemalis (1)

Lophodytes cucullatus (2)

Melanitta fusca deglandi (1)

Melanitta perspicillata $(1,4)$

Mergus merganser (1)

Mergus serrator (1)

Dist: BC, NB, Nfld, Ont

Records: 1. Gibson 1968 (BC); 2. Bain and

Threlfall 1977 (Ont); 3. Mahoney and Threlfall

1978 (Nfld, Ont); 4. Noseworthy and Threlfall
1978 (Ont); 5. Bourgeois and Threlfall 1982

(BC, NB, Nfld)

Streptocara incognita Gibson, 1968

Site: gizzard, under lining

Hosts: Anas platyrhnychos

Bucephala islandica

Oxyura jamaicensis

Dist: Alta, BC

Record: Gibson 1968

Streptocara recta (Linstow, 1879)

Syns: Filaria recta

Spiroptera recta

Site: gizzard, under lining

Host: Podiceps auritus

Dist: BC

Record: Gibson 1968

Streptocara tridentata (Linstow, 1877)

Site: gizzard, under lining

Host: Larus argentatus

Dist: Nfld

Record: Threlfall 1968c

Streptocara species

Site: gizzard, under lining

Hosts: Catoptrophorus semipalmatus inornatus

Charadrius vociferus (2)

Melanitta perspicillata $(1)$

Pluvialis squatarola (2)

Recurvirostra americana (2)

Dist: Alta, BC, NB, Nfld

Records: 1. Bourgeois and Threlfall 1982 (BC,

NB, Nfld); 2. Wong and Anderson unpublished data (Alta)

\section{SUBFAMILY SCHISTOROPHINAE}

Ancyracanthopsis coronata (Molin, 1860)

Syns: Spiroptera coronata

Histiocephalus coronata

Yseria coronata

Skrjabinobronema coronata

Ancyracanthus bilabiatus Molin, 1860

Ancyracanthopsis bilabiatus

Site: gizzard, under lining

Hosts: Actitis macularia $(1,2)$

Limosa fedoa (2)

Dist: Alta, BC, Man

Records: 1. Adams and Gibson 1969 (BC); 2.

Wong and Lankester 1985a (Alta, Man)

Ancyracanthopsis heardi Wong and Anderson, 1990

Site: gizzard, under lining

Host: Catoptrophorus semipalmatus inornatus 
Dist: Alta

Record: Wong and Anderson 1990

Ancyracanthopsis winegardi Wong and Anderson, 1990

Syn: Ancyracanthopsis coronata Wong and

Lankester, 1985

Site: gizzard, under lining

Hosts: Catoptrophorus semipalmatus inornatus

Limosa fedoa

Numenius americanus

Pluvialis squatarola

Dist: Alta, Man

Record: Wong and Anderson 1990

Schistorophus skrjabini (Vassilkova, 1926)

Syn: Antennocara skrjabini

Site: gizzard, under lining

Hosts: Catoptrophorus semipalmatus inornatus

$(1,2)$

Numenius americanus (2)

Dist: Alta, Man

Records: 1. Wong and Lankester 1984a (Man);

2. Wong and Anderson unpublished data (Alta, Man)

Sciadiocara bihamata (Mueller, 1897)

Syns: Ancyracanthus bihamata

Ancyracanthopsis bihamata

Schistorophus bihamatus

Sciadiocara umbellifera Sobolev, 1949

Sciadiocara denticulata Gibson, 1972

Site: gizzard, under lining

Hosts: Actitis macularia $(1,2)$

Catoptrophorus semipalmatus inornatus (3)

Limosa fedoa (3)

Pluvialis squatarola (3)

Dist: Alta, BC, Man

Records: 1. Gibson 1972 (BC); 2. Wong and Lankester 1985b (Man); 3. Wong and Anderson unpublished data (Alta, Man)

Sciadiocara umbellifera (Molin, 1860)

Syns: Spiroptera umbellifera

Schistorophus umbellifera

Spiroptera tantali rubri Molin, 1860

Spiroptera totani Molin, 1860

Site: gizzard, under lining

Hosts: Catoptrophorus semipalmatus inornatus

$(2,3)$

Limosa fedoa (3)

Numenius americanus (3)

Pluvialis squatarola $(1,3)$

Dist: Alta, Man, Que

Records: 1. Gibson 1972 (Que); 2. Wong and Lankester 1985b (Man); 3. Wong and Anderson unpublished data (Alta)
Sobolevicephalus lichtenfelsi Wong and Lankester, 1985

Site: gizzard, under lining

Hosts: Catoptrophorus semipalmatus inornatus $(1,2)$

Limosa fedoa $(1,2)$

Dist: Alta, Man

Records: 1. Wong and Lankester 1985c (Alta,

Man); 2. Wong and Anderson unpublished data (Alta, Man)

Viktorocara capillaris (Molin, 1860)

Syns: Spiroptera capillaris

Cheilospirura capillaris

Schistorophus capillaris

Viktorocara schejkini Guschanskaya, 1950

Site: gizzard, under lining

Hosts: Catoptrophorus semipalmatus inornatus

(3)

Charadrius semipalmatus $(2,3)$

Limosa fedoa $(2,3)$

Pluvialis squatarola $(1,3)$

Dist: Alta, Man, Ont, Que

Records: 1. Gibson 1972 (Que); 2. Wong and

Lankester 1984b (Man); 3. Wong and Anderson unpublished data (Alta, Ont)

Viktorocara limosae Daiya, 1966

Syns: Viktorocara halcyoni Rhizhikov and

Khokhlova, 1964

Viktorocara numenii Petter, 1967

Viktorocara limosae Mawson, 1968

Viktorocara torea Clark, 1978

Site: gizzard, under lining

Hosts: Calidris alpina (1)

Calidris pusilla (4)

Catoptrophorus semipalmatus inornatus $(3,4)$

Charadrius semipalmatus (3)

Limosa fedoa $(3,4)$

Pluvialis squatarola $(2,4)$

Recurvirostra americana (3)

Dist: Alta, BC, Man, NB, Que

Records: 1. Adams and Gibson 1969 (BC); 2.

Gibson 1972 (Que); 3. Wong and Lankester 1984b

(Alta, Man); 4. Wong and Anderson unpublished

data (Alta, Man, NB)

\section{SUPERFAMILY DIPLOTRIAENOIDEA FAMILY DIPLOTRIAENIDAE \\ SUBFAMILY DICHEILONEMATINAE}

Hamatospiculum species

Site: orbit

Host: Asio flammeus

Dist: Que

Record: Mawson 1957 
Monopetalonema alcedinis (Rudolphi, 1819)

Site: air sacs

Host: Ceryle alcyon

Dist: Ont, Que

Records: 1. Mawson 1957 (Que); 2. Anderson 1959b (Ont); 3. Scott 1984 (Que)

Serratospiculoides amaculatum (Wehr, 1938)

Syn: Serratospiculum amaculata

Site: air sacs

Host: Falco mexicanus

Dist: Alta, Man

Records: 1. Bigland et al. 1964 (Alta); 2.

Mawson 1957 (Man)

Dicheilonematid immature worms

Site: air sacs

Host: Puffinus gravis

Dist: Nfld

Record: Bourgeois and Threlfall 1979

\section{SUBFAMILY DIPLOTRIAENINAE}

Diplotriaena agelaius (Walton, 1927)

Syn: Diplotriaenoides translucidus Anderson, 1956

Site: air sacs

Hosts: Dendroica magnolia (3)

Dendroica pensylvanica (3)

Quiscalus quiscula (3)

Seiurus aurocapillus $(1,2)$

Vermivora ruficapilla (3)

Wilsonia canadensis (3)

Dist: Ont

Records: 1. Anderson 1956a; 2. Anderson

1957a; 3. Anderson 1961c

Diplotriaena andersoni Olsen and Braun, 1971

Site: air sacs

Host: Lagopus leucurus

Dist: Alta

Record: Olsen and Braun 1971

Diplotriaena bargusinica Skrjabin, 1917

Syn: Diplotriaena isabellina Koroliova, 1926

Site: air sacs

Hosts: Agelaius phoeniceus (3)

Catharus fuscescens $(1,2)$

Catharus ustulatus (1)

Quiscalus quiscula (1)

Dist: Man, Ont

Records: 1. Anderson 1961c (Ont); 2.

Anderson 1962 (Ont); 3. Hood and Welch 1980

(Man)
Diplotriaena darnaudii Webster and Speckman, 1976

Site: air sacs

Host: Trachyphonus darnaudii

Dist: Man (zoo)

Record: Webster and Speckman 1976

Diplotriaena obtusa (Rudolphi, 1802)

Site: air sacs

Hosts: Progne subis (2)

Dist: Ont

Riparia riparia (1)

Records: 1. Anderson 1961c; 2. Webster 1974

Diplotriaena ozouxi Henry and Ozoux, 1909

Site: air sacs

Hosts: Pooecetes gramineus (2)

Vermivora ruficapilla $(1,3)$

Dist: Ont

Records: 1. Judd 1959 (see Anderson 1959c);

2. Anderson 1959c; 3. Anderson 1961c

Diplotriaena pungens (Schneider, 1866)

Syn: Diplotriaena sialiae Mawson, 1957

Site: air sacs

Hosts: Sialia currucoides (1)

Quiscalus quiscula (2)

Dist: Alta, Man

Records: 1. Mawson 1957 (Alta); 2. Hodasi 1963 (Man)

Diplotriaena sylvinae Pinto and Noronha, 1970

Site: air sacs

Hosts: Tyrannus tyrannus

Tyrannus verticalis

Dist: Man

Record: MacKenzie et al. 1979

Diplotriaena thomasi Siebert, 1944

Site: air sacs

Hosts: Junco hyemalis (1)

Xanthocephalus xanthocephalus (2)

Zonotrichia albicollis (1)

Zonotrichia leucophrys (1)

Dist: Man, Ont

Records: 1. Anderson 1959c (Ont); 2. Hodasi 1963 (Man)

Diplotriaena tricuspis (Fedtschenko, 1874)

Site: air sacs

Hosts: Corvus brachyrhynchos (2)

Dist: Ont

Cyanocitta cristata (1)

Records: 1. Anderson 1961c; 2. Cawthorn and Anderson 1980a, 1980b 
Diplotriaena utae Wong, Anderson and Frimeth, 1983

Site: air sacs

Host: Perisoreus canadensis

Dist: Ont

Record: Wong et al. 1983

Diplotriaena species

Site: air sacs

Hosts: Agelaius phoeniceus (1)

Corvus brachyrhynchos (2)

Quiscalus quiscula (1)

Vermivora ruficapilla (1)

Dist: Ont, Que

Records: 1. Anderson 1957a (Ont); 2. Mawson 1957 (Que)

Quadriplotriaena hypsokysta Crites, 1964

Site: air sacs

Hosts: Sturnella neglecta

Dist.: Alta

Record: Anderson 1989

\section{SUPERFAMILY APROCTOIDEA \\ FAMILY APROCTIDAE \\ SUBFAMILY APROCTINAE}

Aprocta turgida Stossich, 1902

Site: nasal and orbital cavities

Hosts: Larus californicus

Larus delawarensis

Dist: Alta

Record: Vermeer 1969

Pseudaprocta samueli Bartlett and Anderson, 1986

Site: sir sacs

Host: Cyanocitta cristata

Dist: Ont

Record: Bartlett and Anderson 1986

\section{FAMILY DESMIDOCERCIDAE}

Desmidocerca incognita Solonitzin, 1932

Site: air sacs

Host: Phalacrocorax auritus

Dist: NB

Record: Mawson 1957

Desmidocercella numidica (Seurat, 1920)

Syn: Desmidocercella nudicauda Mawson, 1957

Site: air sacs

Hosts: Ardea herodias $(1,2,3)$

Pluvialis squatarola (3)

Dist: BC, NB, Ont, Que
Records: 1. Mawson 1957 (Ont, Que); 2.

Anderson 1959a (Ont); 3. Gibson 1972 (BC, NB, Que)

\section{SUPERFAMILY FILARIOIDEA \\ FAMILY ONCHOCERCIDAE SUBFAMILY DIROFILARIINAE}

Pelecitus fulicaeatrae (Diesing, 1861)

Site: among tendons and muscles near ankle joint

Hosts: Fulica americana (2)

Podiceps auritus (1)

Podiceps grisegena (2)

Podiceps nigricollis (1)

Dist: Alta

Records: 1. Vanderburgh et al. 1984; 2. Bartlett and Anderson 1987c

Pelecitus tubercauda Vanderburgh, Anderson and Stock, 1984

Site: among tendons and muscles of knee region

Host: Geothlypis trichas

Dist: Ont

Record: Vanderburgh et al. 1984

\section{SUBFAMILY LEMDANINAE}

Eufilaria longicaudata Hibler, 1964

Site: connective tissue along trachea and oesophagus

Host: Corvus brachyrhynchos

Dist: Ont

Record: Bartlett and Anderson, 1980a

Eufilaria mcintoshi Anderson and Bennett, 1960

Site: subcutaneous tissue of leg region

Host: Padda oryzivora (imported)

Dist: Ont

Record: Anderson and Bennett 1960

Eufilaria sturninus Bartlett, 1982

Site: connective tissue along trachea and oesophagus

Host: Sturnus vulgaris

Dist: Ont

Record: Bartlett 1982

Eulimdana asperum Bartlett, Anderson and Bush, 1989

Site: connective tissue along trachea and oesophagus

Host: Phalaropus tricolor

Dist: Alta, Man

Record: 1. Bartlett et al. 1989 
Eulimdana florencae Bartlett and Anderson, 1990

Site: connective tissue along trachea and oesophagus

Host: Calidris himantopus

Dist: Alta, Sask

Record: Bartlett and Anderson 1990

Eulimdana juventarum Bartlett, Anderson and

Bush, 1989

Site: connective tissue along trachea and oesophagus

Host: Catoptrophorus semipalmatus inornatus

Dist: Alta, Man

Record: Bartlett et al. 1989

Eulimdana pseudolari Bartlett, Anderson and Bush, 1989

Site: connective tissue along trachea and oesophagus

Host: Phalaropus lobatus

Dist: NB

Record: Bartlett et al. 1989

\section{Eulimdana species}

Site: connective tissue along trachea and oesophagus

Hosts: Calidris alba

Calidris bairdii

Calidris pusilla

Chlidonias niger

Larus pipixcan

Limosa fedoa

Recurvirostra americana

Sterna hirundo

Dist: Alta, NS, Sask

Record: Bartlett unpublished data

Lemdana wernaarti Barltett and Anderson, 1987

Site: connective tissue along trachea and oesophagus

Hosts: Asio otus

Dist: Ont

$$
\text { Bubo virginianus }
$$

Record: Bartlett and Anderson 1987a

Sarconema eurycerca Wehr, 1939

Site: heart

Hosts: Cygnus buccinator $(1,4)$

Cygnus columbianus $(2,3,4)$

Dist: BC, Ont

Records: 1. Cowan 1946 (BC); 2. MacNeill 1975 (BC); 3. Irwin 1975 (Ont); 4. MacNeill and Barnard 1978 (BC)

Sarconema similar to S. eurycerca

Site: heart

Host: Chen caerulescens
Dist: unspecified

Record: Wobeser 1981

Sarconema species

Site: "air sacs"

Host: Melanitta perspicillata

Dist: BC, NB, Nfld

Record: Bourgeois and Threlfall 1982

\section{SUBFAMILY SPLENDIDOFILARIINAE}

Aproctella stoddardi Cram, 1931

Site: body cavity

Hosts: Bonasa umbellus (1)

Catharus fuscescens (3)

Catharus ustulatus (1)

Dendroica coronata (3)

Geothlypis trichas $(1,2)$

Seiurus aurocapillus (2)

Zonotrichia albicollis $(1,2)$

Dist: Ont

Records: 1. Anderson 1957b; Anderson 1961d;

3. Anderson and Freeman 1969

Aproctella species

Site: body cavity

Host: Ceryle alcyon

Dist: Que

Record: Scott 1984

Cardiofilaria pavlovskyi Strom, 1937

Syns: Ornithofilaria inornata Anderson, 1956

Carinema ardea Mawson, 1957

Site: heart, body cavity

Hosts: Accipiter gentilis (6)

Accipiter striatus (3)

Ardea herodias $(2,4)$

Asio otus (3)

Botaurus lentiginosus (2)

Catharus ustulatus (3)

Circus cyaneus (3)

Corvus brachyrhynchos (5)

Corvus corax (3)

Hylocichla mustelina (1)

Icterus galbula (7)

Ixobrychus exilis (4)

Scolopax minor (3)

Seiurus aurocapilus (3)

Sturnus vulgaris (6)

Turdus migratorius (3)

Dist: NB, Ont, Que

Records: 1. Anderson 1956a (Ont); 2. Mawson 1957 (Que); 3. Anderson and Freeman 1969 (Ont); 4. Gibson 1973 (NB, Ont); 5. Bartlett and Anderson 1980a (Ont); 6. Bartlett 1982 (Ont); 7. Vanderburgh and Anderson unpublished data (Ont) 
Chandlerella chitwoodae Anderson, 1961

Syns: Splendidofilaria flexivaginalis Jones, 1961

Chandlerella hispanica Lopez-Caballero, 1974

Site: within connective tissue or lymphatics adjacent to blood vessels in lungs and legs and beside spleen and kidneys

Hosts: Bonasa umbellus (2)

Corvus brachyrhnchos (3)

Dendragapus obscurus (2)

Padda oryzivora (1) (imported)

Dist: BC, Ont

Records: 1. Anderson 1961b (Ont, imported);

2. Gibson 1965 (BC); 3. Bartlett and Anderson 1980a, 1980b (Ont)

Chandlerella bushi Bartlett and Anderson, 1987

Site: within lymphatics adjacent to femoral artery

Host: Fulica americana

Dist: Man

Record: Bartlett and Anderson 1987b

Chandlerella quiscali (Linstow, 1904)

Site: beneath pia mater of cerebrum in region of sagittal fissure

Hosts: Corvus brachyrhynchos (1)

Dist: Ont Quiscalus quiscula (2)

Records: 1. Bartlett and Anderson 1980a; 2.

Bartlett unpublished data

Splendidofilaria algonquinensis (Anderson, 1955)

Syns: Ornithofilaria algonquinensis Anderson, 1955

Splendidofilaria passerina Koch and Huizinga, 1971

Site: wall of pulmonary artery

Host: Hirundo rustica

Dist: Ont

Record: Anderson 1955

Splendidofilaria caperata Hibler, 1964

Site: wall of pulmonary artery

Hosts: Agelaius phoeniceus (3)

Asio otus (4)

Ceryle alcyon (4)

Corvus brachyrhynchos (1)

Fulica americana (5)

Hirundo pyrrhonota (6)

Melospiza melodia (4)

Sturnus vulgaris (2)

Turdus migratorius (3)

Quiscalus quiscula (3)

Dist: Alta, Man, Ont

Records: 1. Bartlett and Anderson 1980a (Ont);

2. Bartlett 1982 (Ont); 3. Bartlett and Anderson
1985 (Ont); 4. Bartlett and Anderson 1987a (Ont);

5. Bartlett and Anderson 1987b (Man); 6. Bartlett unpublished data (Alta)

Splendidofilaria fallisensis (Anderson, 1954)

Syn: Ornithofilaria fallisensis

Site: subcutaneous tissue

Hosts: Anas platyrhynchos (syn: Anas boschas domesticus) $(1,2,3)$

Dist: Ont

Anas rubripes (2)

Records: 1. Anderson 1954; 2. Anderson

1956b; 3. Anderson 1960

Splendidofilaria pectoralis Gibson, 1967

Site: subcutaneous tissue of pectoral region, generally midway between crop and tip of sternum

Hosts: Bonasa umbellus

Dendragapus canadensis

Dendragapus obscurus

Tympanuchus phasianellus

Dist: BC

Record: Gibson 1967

Splendidofilaria wehri Anderson, 1961

Site: behind valve in heart

Hosts: Catharus ustulata (1)

Corvus brachyrhynchos (2)

Dist: Ont

Records: 1. Anderson 1961a; 2. Bartlett and Anderson 1980a

Splendidofilaria species

Site: behind valve in heart

Host: Asio otus

Dist: Ont

Record: Bartlett and Anderson 1987a 


\section{ORDER GAVIIFORMES}

\section{FAMILY GAVIDAE}

Gavia immer (Brunnich)

- Common Loon

- Syn: Colymbus immer Brunnich

Eustrongylides tubifex (NB, Ont)

Gavia stellata (Pont.)

- Red-throated Loon

- Syn: Colymbus stellatus Pont.

Amidostomum cygni (Que)

Gavia species

- Loon

Paracuaria adunca (BC)

\section{ORDER PODICIPEDIFORMES}

\section{FAMILY PODICIPEDIDAE}

Aechmophorus occidentalis (Lawrence)

- Western Grebe

- Syn: Podiceps occidentalis Lawrence

Capillaria obsignata (Alta)

Contracaecum ovale (Alta)

Podiceps auritus (L.)

- Horned Grebe

- Syn: Colymbus auritus L.

Capillaria obsignata (Alta)

Contracaecum ovale (Alta)

Paracuaria adunca (BC)

Pelecitus fulicaecatrae (Alta)

Streptocara recta $(\mathrm{BC})$

Podiceps grisegena (Boddaert)

- Red-necked Grebe

- Syn: Colymbus grisegena Boddaert

Capillaria obsignata (Alta)

Contracaecum ovale (Alta)

Pelecitus fulicaecatrae (Alta)

Podiceps nigricollis Brehm

- Eared Grebe

Capillaria obsignata (Alta)

Contracaecum ovale (Alta)

Pelecitus fulicaecatrae (Alta)

Podilymbus podiceps (L.)

- Pied-billed Grebe

- Syn: Colymbus podiceps L.
Contracaecum podicipitis (unspecified)

Contracaecum spiculigerum (Nfld)

\section{ORDER PROCELLARIIFORMES}

\section{FAMILY DIOMEDEIDAE}

Diomedea nigripes Audubon

- Black-footed Albatross

Paracuaria adunca (BC)

Diomedea species

- Albatross

Contracaecum magnicollare (BC)

Contracaecum pelagicum (BC)

\section{FAMILY PROCELLARIIDAE}

Puffinus gravis (O’Reilly)

- Greater Shearwater

- Syn: Procellaria gravis O'Reilly

Contracaecum species (Nfld)

Dicheilonematid immature worms (Nfld)

Seuratia shipleyi (Nfld)

Stegophorus species (Nfld)

Tetrameres species (NB, Nfld)

\section{ORDER PELECANIFORMES}

\section{FAMILY PHALACROCORACIDAE}

Phalacrocorax auritus (Lesson)

- Double-crested Cormorant

- Syn: Carbo auritus Lesson

Contracaecum spiculigerum (NB)

Desmidocercella incognita (NB)

Syncuaria squamata (Ont)

\section{ORDER CICONIIFORMES SUBORDER ARDEAE}

\section{FAMILY ARDEIDAE}

Ardea herodias L.

- Great Blue Heron

Avioserpens nana (Que)

Cardiofilaria pavlovskyi (NB, Ont, Que)

Desmidocercella numidica (BC, NB, Ont, Que)

Desportesius triaenucha (Que)

Eustrongylides tubifex (NB, Ont)

Microtetrameres canadensis (Que)

Paracuaria adunca (BC)

Tetrameres ardamericanus (Ont) 
Botaurus lentiginosus (Rackett)

- American Bittern

- Syn: Ardea lentiginosa Rackett

Avioserpens species (Ont, Que)

Cardiofilaria pavlovskyi (Que)

Contracaecum microcephalum (Nfld)

Desportesius triaenucha (Ont, Que)

Synhimantus species (Nfld)

Ixobrychus exilis (Gmelin)

- Least Bittern

- Syn: Ardea exilis Gmelin

Avioserpens species (Ont, Que)

Cardiofilaria pavlovskyi (NB, Ont)

\section{ORDER PHOENICOPTERIFORMES}

FAMILY PHOENICOPTERIDAE

Phoenicopterus ruber $\mathrm{L}$.

- Greater Flamingo

Tetrameres occinea $(\mathrm{Nfld})$

\section{ORDER ANSERIFORMES SUBORDER ANSERES}

\author{
FAMILY ANATIDAE \\ SUBFAMILY ANSERINAE
}

Anser anser (L.)

- Graylag Goose

Trichostrongylus tenuis (Ont)

Branta canadensis (L.)

- Canada Goose

- Syn: Anas canadensis L.

Amidostomum anseris (BC, Ont)

Cyathostoma bronchialis (Ont)

Echinuria parva (Ont)

Branta species

- Goose

Amidostomum anseris (Ont)

Echinuria parva (Ont)

Chen caerulescens $(\mathrm{L}$.

- Snow Goose

- Syns: Anas caerulescens L.

Anser hyperboreus Pallas

Amidostomum anseris (Ont)

Amidostomum spatulatum (Ont)

Amidostomum species (BC)

Epomidiostomum crami (Ont)
Sarconema similar to $S$. eurycerca (unspecified)

Trichostrongylus tenuis (NWT)

Chen caerulescens atlanticus (Kennard)

- Greater Snow Goose

Amidostomum spatulatum (unspecified)

Trichostrongylus tenuis (NWT)

Cygnus buccinator Richardson

- Trumpeter Swan

Amidostomum anseris (BC)

Amidostomum species (BC)

Echinuria uncinata (Man)

Sarconema eurycerca (BC)

Cygnus columbianus Ord

- Tundra Swan

- Syn: Anas columbianus Ord

Amidostomum anseris (BC)

Amidostomum cygni (Que)

Amidostomum species (BC)

Sarconema eurycerca (BC, Ont)

Cygnus species

- Swan

Amidostomum anseris (unspecified)

"Ducks, geese and swans"

Acuaria species (Ont)

Amidostomum species (Ont)

Capillaria species (Ont)

Cyathostoma species (Ont)

Tetrameres species (Ont)

"Geese"

Amidostomum anseris (Ont)

"Gosling"

Amidostomum anseris (BC)

"Wild swans"

Amidostomum species (BC)

SUBFAMILY ANATINAE

Anas acuta L.

- Northern Pintail

Amidostomum anseris (Man)

Echinuria uncinata (Man)

Epomidiostomum uncinatum (Man)

Streptocara crassicauda (Man)

Anas clypeata $\mathrm{L}$.

- Northern shoveler

- Syn: Spatula clypeata (L.)

Amidostomum species (Man) 
Epomidiostomum uncinatum (Man)

Streptocara c. crassicauda (BC)

Anas crecca L.

- Green-winged Teal

Amidostomum acutum (NB, NS)

Epomidiostomum uncinatum (NB, NS)

Tetrameres ryjikovi (Nfld)

Anas discors $\mathrm{L}$.

- Blue-winged Teal

Amidostomum acutum (NB, NS)

Amidostumum species (Man)

Capillaria contorta (Que)

Echinuria uncinata (Man)

Epomidiostomum uncinatum (Man, NB, NS)

Streptocara crassicauda (Nfld)

Tetrameres ryjikovi (Nfld)

Anas platyrhynchos $\mathrm{L}$.

- Domestic Duck

Amidostomum anseris (Man, Ont)

Capillaria species (Que)

Splendidofilaria fallisensis (Ont)

Tetrameres crami (Ont, Que)

Anas platyrhynchos $\mathrm{L}$.

- mallard

Echinuria uncinata (Alta, Man)

Epomidiostomum uncinatum (Man)

Streptocara californica (BC)

Streptocara crassicauda (Man)

Streptocara c. crassicauda (BC)

Streptocara formosensis (BC)

Streptocara incognita (Alta, BC)

Anas rubripes Brewster

- American Black Duck

Amidostomum acutum (NB, Nfld, Ont)

Capillaria anatis (NB, Nfld, Ont, Que)

Capillaria obsignata (NB, Nfld)

Capillaria spinulosa (NB, Nfld)

Capillaria species (NB, Nfld, Ont, Que)

Contracaecum anasi (Que)

Epomidiostomum uncinatum (NB, Nfld)

Splendidofilaria fallisensis (Ont)

Tetrameres fissispina (NB, Nfld)

Tetrameres species (unspecified)

Anas strepera $\mathrm{L}$.

- Gadwall

Epomidiostomum uncinatum (Man)

Aythya americana (Eyton)

- Redhead

- Syn: Fuligula americana Eyton

Echinuria uncinata (Man)
Aythya collaris (Donovan)

- Ring-necked Duck

- Syn: Anas collaris Donovan

Capillaria anatis (Ont)

Epomidiostomum crami (NB, Ont)

Streptocara formosensis (Ont)

Tetrameres fissispina (Ont)

Tetrameres species (Ont)

Aythya marila (L.)

- Greater Scaup

- Syn: Anas marila L.

Streptocara californica $(\mathrm{BC})$

Streptocara c. crassicauda (BC)

Aythya valisineria (Wilson)

- Canvasback

- Syn: Anas valisineria Wilson

Echinuria uncinata (Man)

Streptocara californica $(\mathrm{BC})$

Streptocara c. crassicauda (BC)

Bucephala albeola (L.)

- Bufflehead

- Syn: Anas albeola L.

Echinuria uncinata (Man)

Streptocara formosensis (BC)

Bucephala clangula (L.)

- Common Goldeneye

- Syn: Anas clangula L.

Amidostomum acutum (NB, Nfld, Ont)

Amidostomum anseris (BC)

Capillaria anatis (NB, Nfld, Ont, Que)

Capillaria mergi (Nfld, Ont)

Capillaria species (Ont, Que)

Epomidiostomum crami (Ont)

Streptocra c. crassicauda (BC)

Streptocara formosensis (BC, Nfld, Ont)

Tetrameres ryjikovi (Nfld, Ont)

Tetrameres species (unspecified)

Bucephala islandica (Gmelin)

- Barrow's Goldeneye

- Syn: Anas islandica Gmelin

Streptocara californica (BC)

Streptocara c. crassicauda (BC)

Streptocara formosensis (BC)

Streptocara incognita (Alta, BC)

Clangula hyemalis (L.)

- Oldsquaw

- Syn: Anas hyemalis L.

Echinuria borealis (Que)

Streptocara californica (BC)

Streptocara c. crassicauda (BC)

Streptocara formosensis (BC) 
Lophodytes cucullatus (L.)

- Hooded Merganser

- Syn: Mergus cucullatus L.

Capillaria anatis (Ont)

Streptocara crassicauda (Ont)

Streptocara c. crassicauda (BC)

Streptocara formosensis (Ont)

Tetrameres species (Ont)

Melanitta fusca (L.)

- White-winged Scoter

- Syn: Anas fusca L.

Amidostomum acutum (BC, NB, Nfld)

Capillaria nyrocinarum (BC, NB, Nfld)

Streptocara californica (BC, NB, Nfld)

Streptocara crassicauda (BC, NB, Nfld)

Tetrameres fissispina (BC, NB, Nfld)

Tetrameres somateriae (BC, NB, Nfld)

Tetrameres spinosa (BC, NB, Nfld)

Tetrameres species (NB, Nfld, Ont)

Melanitta fusca deglandi (Bonaparte)

- White-winged Scoter

Streptocara formosensis (BC)

Melanitta nigra (L.)

- Black Scoter

- Syn: Anas nigra L.

Amidostomum acutum (BC, NB, Nfld)

Capillaria nyrocinarum (BC, NB, Nfld)

Echinuria hypognatha (BC, NB, Nfld)

Epomidiostomum uncinatum (BC, NB, Nfld)

Streptocara californica (BC, NB, Nfld)

Streptocara crassicauda (BC, NB, Nfld)

Streptocara c. crassicauda $(\mathrm{BC})$

Tetrameres fissispina (BC, NB, Nfld)

Tetrameres somateriae (BC, NB, Nfld)

Tetrameres spinosa (BC, NB, Nfld)

Melanitta perspicillata (L.)

- Surf Scoter

- Syn: Anas perspicillata $\mathrm{L}$.

Amidostomum acutum (BC, NB, Nfld)

Amidostomum species (BC, NB, Nfld)

Capillaria anatis (BC, NB, Nfld)

Capillaria nyrocinarum (BC, NB, Nfld)

Echinuria borealis (BC, NB, Nfld)

Echinuria hypognatha (BC, NB, Nfld)

Paracuaria adunca (BC)

Sarconema species (BC, NB, Nfld)

Streptocara californica (BC, NB, Nfld)

Streptocara crassicauda (BC, NB, Nfld)

Streptocara c. crassicauda (BC)

Streptocara formosensis (BC, Ont)

Streptocara species (BC, NB, Nfld)

Tetrameres fissispina (BC, NB, Nfld)

Tetrameres somateriae (BC, NB, Nfld)
Tetrameres spinosa (BC, NB, Nfld)

Tetrameres species (NB, Nfld, Ont)

"Mergansers"

Eustrongylides mergorum (BC)

Mergus merganser $\mathrm{L}$.

- Common Merganser

Contracaecum yamaguti (unspecified)

Eustrongylides tubifex (NB, Ont)

Streptocara californica (BC)

Streptocara c. crassicauda $(\mathrm{BC})$

Streptocara formosensis (BC)

Mergus serrator $\mathrm{L}$.

- Red-breasted Merganser

Eustrongylides tubifex (NB, Ont)

Streptocara californica $(\mathrm{BC})$

Streptocara c. crassicauda (BC)

Streptocara formosensis (BC)

Oxyura jamaicensis (Gmelin)

- Ruddy Duck

- Syn: Anas jamaicensis Gmelin

Streptocara c. crassicauda (BC)

Streptocara incognita (Alta, BC)

Somateria mollissima (L.)

- Common Eider

- Syn: Anas mollissima L.

Amidostomum acutum (Nfld)

Echinuria borealis (Nfld)

Echinuria uncinata (Nfld)

Paracuaria adunca (Nfld)

Tetrameres somateriae (Nfld)

Somateria spectabilis (L.)

- King Eider

- Syn: Anas spectabilis L.

Amidostomum cygni (Que)

Echinuria borealis (Que)

ORDER FALCONIFORMES SUBORDER ACCIPITRES

\author{
FAMILY ACCIPITRIDAE \\ SUBFAMILY ACCIPITRINAE
}

Accipiter gentilis (L.)

- Northern Goshawk

- Syn: Falco gentilis L.

Cardiofilaria pavlovskyi (Ont)

Accipiter striatus Vieillot

- Sharp-shinned Hawk

Cardiofilaria pavlovskyi (Ont) 
Buteo jamaicensis (Gmelin)

- Red-tailed Hawk

- Syn: Falco jamaicensis Gmelin

Habronema mansioni (Que, Sask)

Circus cyaneus (L.)

- Northern Harrier

- Syn: Falco cyaneus L.

Cardiofilaria pavlovskyi (Ont)

\section{SUBORDER FALCONES}

\section{FAMILY FALCONIDAE}

Falco mexicanus Schlegel

- Prairie Falcon

- Serratospiculoides amaculatum (Alta, Man)

Falco peregrinus anatum Bonaparte

- Peregrine Falcon

Habronema mansioni (Que, Sask)

Porrocaecum trichuriforme (Ont)

\section{ORDER GALLIFORMES}

\section{SUPERFAMILY PHASIANOIDEA \\ FAMILY PHASIANIDAE SUBFAMILY PHASIANINAE}

Phasianus colchicus L.

- Ring-necked Pheasant

Capillaria species (Que)

Heterakis isolonche (Ont)

Syngamus trachea (Ont, Que)

\section{SUBFAMILY TETRAONINAE}

Bonasa umbellus (L.)

- Ruffed Grouse

- Syn: Tetrao umbellus L.

Aproctella stoddardi (Ont)

Ascaridia galli (Ont)

Chandlerella chitwoodae (BC)

Cheilospirura spinosa (Ont)

Oxyspirura petrowi (Ont, Sask)

Splendidofilaria pectoralis (BC)

Centrocercus urophasianus (Bonaparte)

- Sage Grouse

- Syn: Tetrao urophasianus Bonaparte

Oxyspirura petrowi (Ont, Sask)

Dendragapus canadensis (L.)

- Spruce Grouse

- Syn: Tetrao canadensis L.

Splendidofilaria pectoralis (BC)
Dendragapus obscurus (Say)

- Blue Grouse

- Syn: Tetrao obscurus Say

Chandlerella chitwoodae (BC)

Splendidofilaria pectoralis (BC)

Dendragapus obscurus fuliginosus (Ridgway)

- Blue Grouse

Molinacuaria bendelli (BC)

Gallus gallus (L.)

- Domestic Chicken

Ascaridia galli (Man)

Capillaria columbae (Man)

Heterakis gallinarum (Man, Sask)

Lagopus lagopus (L.)

- Willow Ptarmigan

- Syn: Tetrao lagopus L.

Ascaridia compar (Nfld)

Lagopus leucurus (Richardson)

- White-tailed Ptarmigan

- Syn: Tetrao leucurus Richardson

Diplotriaena andersoni (Alta)

Tympanuchus cupido (L.)

- Greater Prairie-chicken

- Syn: Tetrao cupido L.

Acuaria spinosa (Ont)

Oxyspirura petrowi (Ont, Sask)

Tympanuchus phasianellus (L.)

- Sharp-tailed Grouse

- Syns: Pedioecetes phasianellus L. Tetrao phasianellus L.

Cheilospirura spinosa (Ont)

Oxyspirura petrowi (Ont, Sask)

Splendidofilaria pectoralis (BC)

Streptocara c. crassicauda (BC)

SUBFAMILY MELEAGRIDINAE

Meleagris gallopavo L.

- Domestic Turkey

Ascaridia species (Ont)

Ascarididae, unidentified larvae (Man)

SUBFAMILY ODONTOPHORINAE

Callipepla californica (Shaw)

- California Quail

- Syns: Lophortyx californica (Shaw)

Tetrao californica Shaw

Cheilospirura spinosa (BC) 
FAMILY RALLIDAE

SUBFAMILY RALLINAE

Fulica americana Gmelin

- American Coot

Chandlerella bushi (Man)

Pelecitus fulicaeatrae (Alta)

Splendidofilaria caperata (Man)

\section{ORDER CHARADRIIFORMES SUBORDER CHARADRII}

\section{FAMILY CHARADRIIDAE SUMBFAMILY CHARADRIINAE}

Anitibyx armatus (Burchell)

- Blacksmith Plover

Geopetitia aspiculata (Man)

Charadrius semipalmatus Bonaparte

- Semipalmated Plover

- Syn: Tringa hiaticula Ord

Skrjabinoclava pusillae (Alta, Man, NB, NS,

Ont, Sask)

Skrjabinoclava unidentified larvae (Alta, Man,

NB, NS, Ont, Sask)

Stellocaronema skrjabini (Alta, Man)

Viktorocara capillaris (Alta, Man, Ont)

Viktorocara limosae (Alta, Man)

Charadrius vociferus $\mathrm{L}$.

- Killdeer

Chevreuxia americana (Alta, Man)

Dispharynx nasuta (Alta)

Skrjabinocerca prima (Alta)

Streptocara species (Alta)

Viktorocara capillaris (Alta, Man, Ont)

Pluvialis squatarola (L.)

- Black-bellied Plover

- Syn: Tringa squatarola L.

Ancyracanthopsis winegardi (Alta, Man)

Chevreuxia americana (Alta, Man)

Desmidocercella numidica (BC, NB, Que)

Sciadiocara bihamata (Alta, Man)

Sciadiocara umbellifera (Alta, Que)

Skrjabinocerca prima (Alta)

Skrjabinoclava bartlettae (Alta)

Streptocara species (Alta)

Viktorocara capillaris (Alta, Que)

Viktorocara limosae (Alta, Man, Que)
Recurvirostra americana Gmelin

- American Avocet

Chevreuxia americana (Alta, Man)

Eulimdana species (Alta, NS, Sask)

Skrjabinocerca prima (Alta)

Streptocara species (Alta)

Viktorocara limosae (Alta, Man)

\section{SUBORDER SCOLOPACI}

\section{FAMILY SCOLOPACIDAE SUBFAMILY SCOLOPACINAE}

Actitis macularia (L.)

- Spotted Sandpiper

- Syn: Tringa macularia L.

Ancyracanthopsis coronata (BC, Alta, Man)

Sciadiocara bihamata (BC, Man)

Streptocara c. crassicauda $(\mathrm{BC})$

Bartramia longicauda (Bechstein)

- Upland Sandpiper

- Syn: Tringa longicauda Bechstein

Physocephalus sexalatus third-stage larvae (Alta, Man)

Stellocaronema skrjabini (Alta, NB, Sask)

Calidris alba (Pallas)

- Sanderling

- Syn: Tringa alba Pallas

Echinuria uncinata (Alta, Man, NB, Sask)

Eulimdana species (Alta, NS, Sask)

Skrjabinoclava tupacincai (Man, NB, Sask)

Skrjabinoclava unidentified larvae (Alta, Man, NB, NS, Ont, Sask)

Stellocaronema skrjabini (Alta, BC, Man, NB, Sask)

Calidris alpina $(\mathrm{L}$.

- Dunlin

- Syn: Tringa alpina $\mathrm{L}$.

Skrjabinoclava tupacincai (Man, NB, Sask)

Stellocaronema skrjabini (BC, Alta, Man, NB, Sask)

Viktorocara limosae (BC)

Calidris bairdii (Coues)

- Baird's Sandpiper

- Syn: Actodromus bairdii Coues

Echinuria uncinata (Alta)

Eulimdana species (Alta, NS, Sask)

Calidris himantopus (Bonaparte)

- Stilt Sandpiper

- Syn: Tringa himantopus Bonaparte 
Eulimdana florencae (Alta, Sask)

Stellocaronema skrjabini (Alta, NB, Sask)

Calidris mauri (Cabanis)

- Western Sandpiper

- Syn: Ereunetes mauri Cabanis

Stellocaronema skrjabini (BC)

Calidris minutilla (Vieillot)

- Least Sandpiper

- Syn: Tringa minutilla Vieillot

Stellocaronema skrjabini (Alta, NB, Sask)

Calidris pusilla (L.)

- Semipalmated Sandpiper

- Syn: Tringa pusilla L.

Echinuria uncinata (Alta, Man, NB, Sask)

Eulimdana species (Alta, NS, Sask)

Skrjabinoclava bakeri (Alta, NB, NS, Ont)

Skrjabinoclava morrisoni (Alta, Man, NB, NS,

Ont, Sask)

Skrjabinoclava pusillae (Alta, Man, NB, NS,

Ont, Sask)

Skrjabinoclava tupacincai (Man, NB, Sask)

Skrjabinoclava unidentified larvae (Alta, Man,

NB, NS, Ont, Sask)

Stellocaronema skrjabini (Alta, NB, Sask)

Viktorocara limosae (Alta, Man, NB)

Catoptrophorus semipalmatus inornatus (Brewster)

- Western Willet

Ancyracanthopsis heardi (Alta)

Ancyracanthopsis winegardi (Alta, Man)

Cosmocephalus obvelatus (Alta)

Echinuria uncinata (Alta, Man)

Eulimdana juventarum (Alta, Man)

Schistorophus skrjabini (Alta, Man)

Sciadiocara bihamata (Alta, BC, Man)

Sciadiocara umbellifera (Alta, Man)

Skrjabinocerca prima (Alta)

Skrjabinoclava inornatae (Alta, Man)

Sobolevicephalus lichtenfelsi (Alta, Man)

Stellocaronema skrjabini (Alta, Man, NB, Sask)

Streptocara species (Alta)

Tetrameres species (Alta)

Viktorocara capillaris (Alta, Ont)

Viktorocara limosae (Alta, Man)

Gallinago gallinago (L.)

- Common Snipe

- Syns: Capella gallinago (L.)

Scolopax gallinago $\mathrm{L}$.

Capillaria contorta (Nfld)

Cosmocephalus capellae (Ont)

Limnodromus scolopaceus (Say)

- Long-billed Curlew
- Syn: Limosa scolopacea Say

Stellocaronema skrjabini (Alta)

Limosa fedoa (L.)

- Marbled Godwit

- Syn: Scolopax fedoa L.

Ancyracanthopsis coronata (Alta, Man)

Ancyracanthopsis winegardi (Alta, Man)

Cosmocephalus obvelatus (Alta)

Echinuria uncinata (Alta, Man)

Eulimdana species (Alta, NS, Sask)

Sciadiocara bihamata (Alta, Man)

Sciadiocara umbellifera (Alta)

Skrjabinoclava kritscheri (Alta, Man)

Sobolevicephalus lichtenfelsi (Alta, Man)

Stellocaronema skrjabini (Alta, Man)

Viktorocara capillaris (Alta, Man)

Vikotorcara limosae (Alta, Man)

Numenius americanus Bechstein

- Long-billed Curlew

Acuaria species (Alta)

Ancyracanthopsis winegardi (Alta, Man)

Pectinospirura argentata (Alta)

Physocephalus sexalatus third-stage larvae

(Alta, Man)

Schistorophus skrjabini (Alta, Man)

Sciadiocara umbellifera (Alta)

Scolopax minor (Gmelin)

- American Woodcock

Cardiofilaria pavlovskyi (Ont)

Tringa flavipes (Gmelin)

- Lesser Yellowlegs

- Syn: Scolopax flavipes Gmelin

Cosmocephalus obvelatus (Alta)

Tringa melanoleuca (Gmelin)

- Greater Yellowlegs

- Syn: Scolopax melanoleuca Gmelin

Stellocaronema skrjabini (Man)

\section{SUBFAMILY PHALAROPODINAE}

Phalaropus lobatus (L.)

- Red-necked phalarope

- Syn: Tringa lobata L.

Eulimdana pseudolari (NB)

Phalaropus tricolor (Vieillot)

- Wilson's Phalarope

- Syn: Steganopus tricolor Vieillot

Cosmocephalus obvelatus (Alta)

Echinuria uncinata (Alta, Man)

Eulimdana asperum (Alta, Man)

Skrjabinocerca prima (Alta) 


\section{SUBORDER LARI}

\section{FAMILY LARIDAE SUBFAMILY LARINAE}

Larus argentatus Pont.

- Herring Gull

Capillaria contorta (Nfld)

Contracaecum spiculigerum (Nfld)

Contracaecum species (Nfld)

Cosmocephalus obvelatus (Nfld, Que)

Cyathostoma lari (Nfld)

Paracuaria adunca (BC)

Porrocaecum semiteres (Nfld)

Robertdollfusa species (Nfld)

Streptocara tridentata (Nfld)

Larus canus $\mathrm{L}$.

- Mew Gull

Paracuaria adunca (BC)

Larus californicus Lawrence

- California Gull

Aprocta turgida (Alta)

Larus delawarensis Ord

- Ring-billed Gull

Aprocta turgida (Alta)

Capillaria species (Ont)

Cosmocephalus obvelatus (Ont)

Paracuaria adunca (Ont)

Pectinospirura argentata (Ont)

Skrjabinoclava inornata (Ont)

Tetrameres species (Ont)

Larus glaucescens Naumann

- Glaucous-winged Gull

Paracuaria adunca (BC)

Larus marinus $\mathrm{L}$.

- Great Black-backed Gull

Contracaecum spiculigerum (Nfld)

Cosmocephalus obvelatus (Nfld)

Paracuaria adunca (Atlantic coast)

Larus pipixcan Wagler

- Franklin's Gull

Eulimdana species (Alta, NS, Sask)

Paracuaria adunca (midwest)

Larus thayeri Brooks

- Thayer's Gull

Paracuaria adunca (BC)

Streptocara c. crassicauda $(\mathrm{BC})$

Rissa tridactyla $(\mathrm{L}$.

- Black-legged Kittiwake
- Syn: Larus tridactyla L.

Contracaecum spiculigerum (Nfld)

Cosmocephalus obvelatus (Nfld)

SUBFAMILY STERNINAE

Chlidonias niger $(\mathrm{L}$.

- Black Tern

- Syn: Sterna niger L.

Eulimdana species (Alta, NS, Sask)

Sterna hirundo L.

- Common Tern

Eulimdana species (Alta, NS, Sask)

SUBORDER ALCAE

FAMILY ALCIDAE

Alca torda L.

- Razorbill

Contracaecum spiculigerum (Nfld)

Cepphus columba Pallas

- Pigeon Guillemot

Paracuaria adunca (BC)

Streptocara c. crassicauda (BC)

Uria aalge (Pont.)

- Common Murre

- Syn: Colymbus aalge Pont.

Contracaecum spiculigerum (Nfld)

Cosmocephalus obvelatus (Nfld)

Eustrongylides mergorum (Nfld)

Paracuaria adunca (BC)

Uria lomvia (L.)

- Thick-billed Murre

- Syn: Alca lomvia L.

Contracaecum spiculigerum (Nfld)

Eustrongylides mergorum (Nfld)

Stegophorus stellae-pollaris (Nfld)

\section{ORDER COLUMBIFORMES SUBORDER COLUMBAE}

\section{FAMILY COLUMBIDAE}

Columba livia Gmelin

- Rock Dove

- Syn: Columba domestica livia Gmelin

Ornithostrongylus quadriradiatus (Ont)

Columba species

- Pigeon

Ornithostrongylus quadriradiatus (unspecified) 


\section{ORDER STRIGIFORMES}

FAMILY STRIGIDAE

Asio flammeus (Pont.)

- Short-eared Owl

- Syn: Strix flammea Pont.

Hamatospiculum species (Que)

Asio otus (L.)

- Long-eared Owl

- Syn: Strix otus L.

Cardiofilaria pavlovskyi (Ont)

Lemdana wernaarti (Ont)

Splendidofilaria caperata (Ont)

Splendidofilaria species (Ont)

Bubo virginianus (Gmelin)

- Great Horned Owl

- Syn: Strix virginianus Gmelin

Capillaria falconis-nisi (Que)

Cyrnea americanum (Alta)

Lemdana wernaarti (Ont)

Microtetrameres bubo (Alta)

Porrocaecum depressum (Alta, Man)

Porrocaecum trichuriforme (Ont)

Nyctea scandiaca (L.)

- Snowy Owl

- Syn: Strix scandiaca L.

Cyrnea longispicula (Alta)

Microtetrameres canadensis (Que)

Microtetrameres species (Alta)

"Owl"

Capillaria falconis-nisi (Que)

\section{ORDER CORACIIFORMES SUBORDER UPUPAE}

\section{FAMILY PHOENICULIDAE}

Phoeniculus purpureus (Miller)

- Green Wood Hoopoe

Geopetitia aspiculata (Man)

\section{SUPERFAMILY ALCEDINOIDEA \\ FAMILY ALCEDINIDAE \\ SUBFAMILY CERYLINAE}

Ceryle alcyon (L.)

- Belted Kingfisher

- Syn: Alcedo alcyon L.

Aproctella species (Que)

Aviculariella alcyona (Ont, Que)

Capillaria species (Que)
Monopetalonema alcedinis (Ont, Que)

Splendidofilaria caperata (Ont)

Strongyloides species (Que)

Syngamus trachea (Que)

\section{ORDER PICIFORMES} SUBORDER PICI

\section{FAMILY CAPITONIDAE}

Trachyphorus darnaudii (Prevost and Des Murs)

- d'Arnaud's Barbet

Diplotriaena darnaudii (Man)

ORDER PASSERIFORMES

SUBORDER TYRANNI

SUPERFAMILY TYRANNOIDEA

FAMILY TYRANNIDAE

SUBFAMILY TYRANNINAE

Pitangus sulphuratus (L.)

- Great Kiskadee

- Syn: Lanius sulphuratus L.

Geopetitia aspiculata (Man)

Tyrannus tyrannus (L.)

- Eastern Kingbird

- Syn: Lanius tyrannus L.

Acuaria species (Man)

Diplotriaena sylvinae (Man)

Microtetrameres species (Man)

Tyrannus verticalis Say

- Western Kingbird

Acuaria species (Man)

Diplotriaena sylvinae (Man)

\section{SUBORDER PASSERES}

\section{FAMILY HIRUNDINIDAE SUBFAMILY HIRUNDININAE}

Hirundo pyrrhonota Vieillot

- Cliff Swallow

Splendidofilaria caperata (Alta)

Hirundo rustica $\mathrm{L}$.

- Barn Swallow

Splendidofilaria algonquinensis (Ont)

Progne subis (L.)

- Purple Martin

- Syn: Hirundo subis L.

Diplotriaena obtusa (Ont) 
Riparia riparia (L.)

- Bank Swallow

- Syn: Hirundo riparia L.

Diplotriaena obtusa (Ont)

FAMILY CORVIDAE

Corvus brachyrhynchos Brehm

- American Crow

Acuaria anthuris (Ont, Que)

Ascarididae unidentified larvae (Nfld)

Capillaria species (Que)

Cardiofilaria pavlovskyi (Ont)

Chandlerella chitwoodae (Ont)

Chandlerella quiscali (Ont)

Diplotriaena tricuspis (Ont)

Diplotriaena species (Que)

Eufilaria longicaudata (Ont)

Microtetrameres helix (Man, Que)

Porrocaecum ensicaudatum (Que)

Porrocaecum species (Que)

Splendidofilaria caperata (Ont)

Splendidofilaria wehri (Ont)

Corvus corax $\mathrm{L}$.

- Common Raven

Cardiofilaria pavlovskyi (Ont)

Cyanocitta cristata (L.)

- Blue Jay

- Syn: Corvus cristatus L.

Diplotriaena tricuspis (Ont)

Pseudaprocta samueli (Ont)

Perisoreus canadensis (L.)

- Gray Jay

- Syn: Corvus canadensis L.

Diplotriaena utae (Ont)

FAMILY PYCNONOTIDAE

Pycnonotus cafer (L.)

- Red-vented Bulbul

- Syn: Turdus cafer L.

Geopetitia aspiculata (Man)

Pycnonotus jocosus (L.)

- Red-whiskered Bulbul

- Syn: Lanius jocosus L.

Geopetitia aspiculata (Man)

\section{FAMILY IRENIDAE}

Chloropsis hardwikei Jardine and Selby

- Orange-bellied Leafbird

Geopetitia aspiculata (Man)
Irena puella (Latham)

- Blue-backed Fairy Bluebird

Geopetitia aspiculata (Man)

\section{FAMILY MUSCICAPIDAE SUBFAMILY MONARCHINAE}

Sialia currucoides (Bechstein)

- Mountain Bluebird

- Syn: Motacilla currucoides Bechstein

Diplotriaena pungens (Alta)

\section{SUBFAMILY TURDINAE}

Catharus fuscescens (Stephens)

- Veery

- Syn: Turdus fuscescens Stephens

Aproctella stoddardi (Ont)

Diplotriaena bargusinica (Ont)

Catharus ustulatus (Nuttall)

- Swainson's Thrush

- Syn: Turdus ustulatus Nuttall

Aproctella stoddardi (Ont)

Cardiofilaria pavlovskyi (Ont)

Diplotriaena bargusinica (Ont)

Splendidofilaria wehri (Ont)

Hylocichla mustelina (Gmelin)

- Wood Thrush

- Syn: Turdus mustelinus Gmelin

Cardiofilaria pavlovskyi (Ont)

Myiophoneus caeruleus (Scopoli)

- Himalayan Whistling Thrush

Geopetitia aspiculata (Man)

Turdus migratorius L.

- American Robin

Capillaria caudinflata (Que)

Cardiofilaria pavlovskyi (Ont)

Porrocaecum ensicaudatum (Ont, Que)

Porrocaecum species (Que)

Slendidofilaria caperata (Ont)

SUBFAMILY TIMALINAE

Minla cyanouroptera (Hodgson)

- Blue-winged Minla

Geopetitia aspiculata (Man) 
FAMILY BOMBYCILLIDAE

Bombycilla cedrorum Vieillot

- Ceder Waxwing

Cosmocephalus obvelatus (Nfld)

\section{FAMILY STURNIDAE} SUBFAMILY STURNINAE

Sturnus vulgaris $\mathrm{L}$.

- European Starling

Capillaria ovopunctatum (Que)

Cardiofilaria pavlovskyi (Ont)

Eufilaria sturninus (Ont)

Porrocaecum ensicaudatum (Ont)

Splendidofilaria caperata (Ont)

FAMILY EMBERIZIDAE SUBFAMILY PARULINAE

Dendroica coronata (L.)

- Yellow-rumped Warbler

- Syn: Motacilla coronata L.

Aproctella stoddardi (Ont)

Dendroica magnolia (Wilson)

- Magnolia Warbler

- Syn: Sylvia magnolia Wilson

Diplotriaena agelaius (Ont)

Dendroica pensylvanica (L.)

- Chestnut-sided Warbler

- Syn: Motacilla pensylvanica $\mathrm{L}$.

Diplotriaena agelaius (Ont)

Geothlypis trichas (L.)

- Common Yellowthroat

- Syn: Turdus trichas L.

Aproctella stoddardi (Ont)

Pelecitus tubercauda (Ont)

Seiurus aurocapillus (L.)

- Ovenbird

- Syn: Motacilla aurocapilla L.

Aproctella stoddardi (Ont)

Cardiofilaria pavlovskyi (Ont)

Diplotriaena agelaius (Ont)

Seiurus noveboracensis (Gmelin)

- Northern Waterthrush

- Syn: Motacilla noveboracensis Gmelin

Syngamus trachea (Nfld)

Vermivora ruficapilla (Wilson)

- Nashville Warbler

- Syn: Sylvia ruficapilla Wilson

Diplotriaena agelaius (Ont)
Diplotriaena ozouxi (Ont)

Diplotriaena species (Ont)

Wilsonia canadensis (L.)

- Canada Warbler

- Syn: Muscicapa canadensis L.

Diplotriaena agelaius (Ont)

\section{SUBFAMILY THRAUPINAE}

Tachyphonus rufus (Boddaert)

- White-lined Tanager

- Syn: Tangara rufa Boddaert

Geopetitia aspiculata (Man)

Thraupis episcopus (L.)

- Blue-gray Tanager

- Syn: Tanagra episcopus L.

Geopetitia aspiculata (Man)

Thraupis palmarum (Wied)

- Palm Tanager

- Syn: Tanagra palmarum Wied

Geopetitia aspiculata (Man)

\section{SUBFAMILY CARDINALINAE}

Saltator coerulescens Vieillot

- Grayish Saltator

Geopetitia aspiculata (Man)

\section{SUBFAMILY EMBERIZINAE}

Junco hyemalis (L.)

- Dark-eyed Junco

- Syn: Fringilla hyemalis L.

Diplotriaena thomasi (Ont)

Melospiza melodia (Wilson)

- Song Sparrow

- Syn: Fringilla melodia Wilson

Splendidofilaria caperata (Ont)

Passerella iliaca (Merrem)

- Fox Sparrow

- Syn: Fringilla iliaca Merrem

Capillaria contorta (Nfld)

Porrocaecum brevispiculum (Nfld)

Syngamus trachea (Nfld)

Pipilo erythropthalmus (L.)

- Rufous-sided Towhee

- Syn: Fringilla erythropthalmus L.

Capillaria tridens (Man)

Pooecetes gramineus (Gmelin)

- Vesper Sparrow 
- Syn: Fringilla graminea Gmelin

Diplotriaena ozouxi (Ont)

Zonotrichia albicollis (Gmelin)

- White-throated Sparrow

- Syn: Fringilla albicollis Gmelin

Aproctella stoddardi (Ont)

Diplotriaena thomasi (Ont)

Zonotrichia leucophrys (Forster)

- White-crowned Sparrow

- Syn: Emberiza leucophrys Forster

Diplotriaena thomasi (Ont)

\section{SUBFAMILY ICTERINAE}

Agelaius phoeniceus (L.)

- Red-winged Blackbird

- Syn: Oriolus phoeniceus L.

Diplotriaena bargusinica (Man)

Diplotriaena species (Ont)

Dispharynx nasuta (Man, Que)

Microtetrameres species (Man, Que)

Porrocaecum species (Que)

Splendidofilaria caperata (Ont)

Syngamus trachea (Que)

Tetrameres species (Man)

Icterus galbula (L.)

- Northern Oriole

- Syn: Coracias galbula L.

Cardiofilaria pavlovskyi (Ont)

Molothrus ater (Boddaert)

- Brown-headed Cowbird

- Syn: Oriolus ater Boddaert

Porrocaecum ensicaudatum (Que)
Quiscalus quiscula (L.)

- Common Grackle

Chandlerella quiscali (Ont)

Diplotriaena agelaius (Ont)

Diplotriaena bargusinica (Ont)

Diplotriaena pungens (Man)

Diplotriaena species (Ont)

Porrocaecum ensicaudatum (Ont, Que)

Splendidofilaria caperata (Ont)

Quiscalus quiscula versicolor Vieillot

- Bronzed Grackle

Capillaria ovopunctatum (Man)

Microtetrameres species (Man)

Sturnella neglecta Audubon

- Western meadowlark

Quadriplotriaena hypsokysta (Alta)

Xanthocephalus xanthocephalus (Bonaparte)

- Yellow-headed Blackbird

- Syn: Icterus xanthocephalus Bonaparte

Diplotriaena thomasi (Man)

\section{FAMILY FRINGILLIDAE SUBFAMILY FRINGILLINAE}

Padda oryzivora (L.)

- Java Sparrow

- Syn: Loxia oryzivora L.

Eufilaria mcintoshi (Ont)

Chandlerella chitwoodae (Ont) 
ADAMS, J.R. and G.G. GIBSON. 1969. Ancyracanthopsis bendelli $\mathrm{n}$.sp. (Acuariidae: Schistorophinae) from Pacific coast grouse, with observations on related nematode genera. Canadian Journal of Zoology 47: 619-626.

ADDISON, E.M. and R.C. ANDERSON. 1969. Oxyspirura lumsdeni n.sp. (Nematoda: Thelaziidae) from Tetraonidae in North America. Canadian Journal of Zoology 47: 1223-1227.

AMERICAN ORNITHOLOGISTS' UNION. 1983. Check-list of North American birds. 6th Edition. Allen Press, Kansas

ANDERSON, R.C. 1954. Ornithofilaria fallisensis n.sp. (Nematoda: Filarioidea) from the domestic duck with descriptions of microfilariae in waterfowl. Canadian Journal of Zoology 32: 125-137.

\section{ANDERSON, R.C. 1955. Ornithofilaria} algonquinensis $\mathrm{n}$.sp. from Hirundo erythrogaster with a revision of the genera Paramicipsella Chow, 1939 emend. Chabaud and Choquet, 1953 and Ornithofilaria Gonnert, 1937. Canadian Journal of Zoology 33: 107-112.

ANDERSON, R.C. 1956a. Two new filarioid nematodes from Ontario birds. Canadian Journal of Zoology 34: 213-218.

ANDERSON, R.C. 1956b. The life cycle and seasonal transmission of Ornithofilaria fallisensis Anderson, a parasite of domestic and wild ducks. Canadian Journal of Zoology 34: 485-525.

ANDERSON, R.C. 1957a. Observations on the life cycles of Diplotriaenoides translucidus Anderson and members of the genus Diplotriaena. Canadian Journal of Zoology 35: 15-24.

ANDERSON, R.C. 1957b. Taxonomic studies on the genera Aproctella Cram, 1931 and Carinema Pereira and Vaz, 1933 with a proposal for a new genus Pseudaproctella. Canadian Journal of Zoology 35: 25-33.

ANDERSON, R.C. 1959a. The egg and first-stage larva of Desmidocercella numidica (Seurat,
1920) with remarks on the affinities of the Desmidocercidae. Canadian Journal of Zoology 37: 407-413.

ANDERSON, R.C. 1959b. The morphology of Monopetalonema alcedinis (Rudolphi, 1819) (Nematoda: Filarioidea) including its first-stage larva. Canadian Journal of Zoology 37: 609-614.

ANDERSON, R.C. 1959c. Preliminary revision of the genus Diplotriaena Henry and Ozoux, 1909 (Diplotriaenidae: Diplotriaeninae). Parassitologia 1: 195-307.

ANDERSON, R.C. 1960. Correction to a previous paper. Canadian Journal of Zoology 38: 677.

ANDERSON, R.C. 1961a. Splendidofilaria wehri n.sp. with a revision of Splendidofilaria and related genera. Canadian Journal of Zoology 39: 201-207.

ANDERSON, R.C. 1961b. Study of two filarioid nematodes, Chandlerella chitwoodae n.sp. from Padda oryzivora (L.) and Protofilaria furcata Chandler, 1929. Canadian Journal of Zoology 39: 317-323.

ANDERSON, R.C. 1961c. Further observations on Diplotriaena in birds. Canadian Journal of Zoology 39: 377.

ANDERSON, R.C. 1961d. On the identity of Aproctella in birds in North America. Proceedings of the Helminthological Society of Washington 28: 81-82.

ANDERSON, R.C. 1962. On the development, morphology, and experimental transmission of Diplotriaena bargusinica (Filarioidea: Diplotriaenidae). Canadian Journal of Zoology 40: 1175-1186.

ANDERSON, R.C. 1989. The development of Quadriplotriaena hypsokysta (Nematoda: Diplotriaenoidea) in grasshoppers (Orthoptera). Proceeding of the Helminthological Society of Washington 56: 199-201

ANDERSON, R.C. and G.F. BENNETT. 1960. Eufilaria mcintoshi n.sp. from Padda oryzivora (L.). Proceedings of the Helminthological Society of Washington 27: 113-115. 
ANDERSON, R.C., A.G. CHABAUD and S. WILLMOTT. 1974-1983. CIH Keys to the nematode parasites of vertebrates. Nos. 1-10. Commonwealth Agricultural Bureaux, Farnham Royal, Bucks, England.

ANDERSON, R.C. and R.S. FREEMAN. 1969. Cardiofilaria inornata (Anderson, 1956) from woodcock with a review of Cardiofilaria and related genera (Nematoda: Filarioidea). Transactions of the American Microscopical Society 88: 68-79.

ANDERSON, R.C. and P.L. WONG. 1981. Redescription of Cosmocephalus obvelatus (Creplin, 1825) (Nematoda: Acuarioidea) from Larus delawarensis Ord (Laridae). Canadian Journal of Zoology 59: 1897-1902.

ANDERSON, R.C., P.L. WONG and U.R. STRELIVE. 1980. Restudy of the generitype of Aviculariella. A. alcyona Wehr, 1931 (Nematoda: Acuariidae). Systematic Parasitology 1: 105-109.

ANDREWS, S.E. and W. THRELFALL. 1975. Parasites of the common crow (Corvus brachyrhynchos Brehm, 1822) in insular Newfoundland. Proceedings of the Helminthological Society of Washington 42: 24-28.

ANON. 1967. Status of wildlife research projects for the year, 1966. Ontario Department of Lands and Forest Section Report (Wildlife) 65: 1-52.

BAIN, G.A. and W. THRELFALL. 1977. Helminth parasites of hooded mergansers, Lophodytes cucullatus (L.), from Ontario. Proceedings of the Helminthological Society of Washington 44: 219-221.

BARTLETT, C.M. 1982. Filarioid nematodes in the starling (Sturnus v. vulgaris L.) in southern Ontario, Canada, with a description of Eufilaria sturninus $\mathrm{n}$.sp. Canadian Journal of Zoology 60: 2409-2413.

BARTLETT, C.M. and R.C. ANDERSON. 1980a. Filarioid nematodes (Filarioidea: Onchocercidae) of Corvus brachyrhynchos brachyrhynchos Brehm in southern Ontario, Canada and a consideration of the epizootiology of avian filariasis. Systematic Parasitology 2: 77-102.
BARTLETT, C.M. and R.C. ANDERSON. 1980b. Development of Chandlerella chitwoodae Anderson, 1961 (Filarioidea: Onchocercidae) in Culicoides stilobezzioidea Foote and Pratt and C. travisi Vargas (Diptera:

Ceratopogonidae). Canadian Journal of Zoology 58: 1002-1006.

BARTLETT, C.M. and R.C. ANDERSON. 1985. On the filarioid nematodes (Splendidofilaria spp.) from the pulmonary arteries of birds. Canadian Journal of Zoology 63: 2373-2377.

BARTLETT, C.M. and R.C. ANDERSON. 1986. Pseudaprocta samueli sp.n. (Nematoda: Aproctoidea) from the blue jay (Cyanocitta cristata) in Canada, with comments on the genus. Proceedings of the Helminthological Society of Washington 53: 270-276.

BARTLETT, C.M. and R.C. ANDERSON. 1987a. Lemdana wernaarti $\mathrm{n} . \mathrm{sp}$. and other filarioid nematodes from Bubo virginianus and Asio otus (Strigiformes) in Ontario, Canada, with a revision of Lemdana and a key to avian filarioid genera. Canadian Journal of Zoology 65: 1100-1109.

BARTLETT, C.M. and R.C. ANDERSON. 1987b. Chandlerella bushi $\mathrm{n} . \mathrm{sp}$. and Splendidofilaria caperata Hibler, 1964 (Nematoda: Filarioidea) from Fulica americana (Gruiformes: Rallidae) in Manitoba, Canada. Canadian Journal of Zoology 65: 2799-2802.

BARTLETT, C.M. and R.C. ANDERSON. 1987c. Pelecitus fulicaeatrae (Nematoda: Filarioidea) of coots (Gruiformes) and grebes (Podicipediformes): skin-inhabiting microfilariae and development in Mallophaga. Canadian Journal of Zoology 65: 2803-2812.

BARTLETT, C.M. and R.C. ANDERSON. 1990. Eulimdana florencae n.sp. (Nematoda: Filarioidea) from Micropalama himantopus (Aves: Charadriiformes): evidence for neonatal transmission, ephemeral adults, and long-lived microfilariae among filarioids of shorebirds. Canadian Journal of Zoology 68: 986-992.

BARTLETT, C.M., R.C. ANDERSON and A.O. BUSH. 1989. Taxonomic descriptions and comments on the life history of new species of Eulimdana (Nematoda: Filarioidea) with skin-inhabiting microfilariae in the Charadriiformes (Aves). Canadian Journal of Zoology 67: 612-629 
BARTLETT, C.M., R.C. ANDERSON and P.L. WONG. 1989. Development of Skrjabinocerca prima (Nematoda: Acuarioidea) in Hyalella azteca (Amphipoda) and Recurvirostra americana (Aves: Charadriiformes), with comments on its precocity. Canadian Journal of Zoology 67: 2883-2892.

BARTLETT, C.M., A.O. BUSH and R.C. ANDERSON. 1987. Unusual finding of encapsulated nematode larvae (Spiruroidea) in Bartramia longicauda and Numenius americanus (Charadriiformes) in western Canada. Journal of Wildlife Diseases 23: 591-595.

BARTLETT, C.M., G.J. CRAWSHAW and R.G. APPY. 1984. Epizootiology, development, and pathology of Geopetitia aspiculata Webster, 1971 (Nematoda: Habronematoidea) in tropical birds at the Assiniboine Park Zoo, Winnipeg, Canada. Journal of Wildlife Diseases 20: 289-299.

BIGLAND, C.H., S.K. LIU and M.L. PERRY. 1964. Five cases of Serratospiculum amaculata (Nematoda: Filarioidea) infection in prairie falcons (Falco mexicanus). Avian Diseases 8: 412-419.

BISHOP, C.A. and W. THRELFALL. 1974. Helminth parasites of the common eider duck, Somateria mollissima (L.), in Newfoundland and Labrador. Proceedings of the Helminthological Society of Washington 41: 25-35.

BLANCHARD, J.A.G. and C.V. SAINT-JACQUES. 1979. Treatment of an outbreak of Syngamus trachea infection in pheasant with Thiabendazole. MV Quebec 9: 7-10.

BOURGEOIS, C.E. and W. THRELFALL. 1979. Parasites of the greater shearwater (Puffinus gravis) from Newfoundland, Canada. Canadian Journal of Zoology 57: 1355-1357.

BOURGEOIS, C.E. and W. THRELFALL. 1982. Metazoan parasites of three species of scoter (Anatidae). Canadian Journal of Zoology 60: 2253-2257.

BOYD, E.M. 1966. Observations on nematodes of herons in North America including three new species and new host and state records. Journal of Parasitology 52: 503-511.
BOYD, E.M. and A.E. FRY. 1971. Metazoan parasites of the eastern belted kingfisher, Megaceryle alcyon alcyon. Journal of Parasitology 57: 150-156.

BUSCHER, H.N. 1965. Dynamics of the intestinal helminth fauna in three species of ducks. Journal of Wildlife Management 29: 772-781.

BUSCHER, H.N. 1966. Intestinal helminths of the blue-winged teal, Anas discors L., at Delta, Manitoba. Canadian Journal of Zoology 44: 113-116.

CAWTHORN, R.J. and R.C. ANDERSON 1980a. Development of Diplotriaena tricuspis (Nematoda: Diplotriaenoidea), a parasite of Corvidae, in intermediate and definitive hosts. Canadian Journal of Zoology 58: 94-108.

CAWTHORN, R.J. and R.C. ANDERSON. 1980b. Diplotriaena tricuspis (Fedtschenko, 1874)

(Nematoda: Diplotriaenoidea) in crows (Corvus brachyrhynchos Brehm) wintering in Ontario. Journal of Wildlife Diseases 16: 363-365.

CHANDLER, R.E. 1970. Helminth parasites of California quail (Lophortyx californicus) from the Okanagan Valley, British Columbia. Canadian Journal of Zoology 48: 741-744.

CLARKE, C.H.D. 1936. Fluctuations in numbers of ruffed grouse, Bonasa umbellus (Linne), with special reference to Ontario. University of Toronto Studies in Biology Series 41: 1-118.

CORNWELL, G. 1963. Observations on waterfowl mortality in southern Manitoba caused by Echinuria uncinata (Nematoda, Acuariidae). Canadian Journal of Zoology 41: 699-703.

COWAN, I.T. 1946. Death of a trumpeter swan from multiple parasitism. Auk 63: 248-249.

CRAM, E.B. 1928. Nematodes of pathological significance found in some economically important birds in North America. United States Department of Agriculture Technical Bulletin 49: 1-9.

CRICHTON, V. and H. WELCH. 1972. Helminths from the digestive tracts of mallards and pintails in the Delta Marsh, Manitoba. Canadian Journal of Zoology 50: 633-637. 
FERNANDO, M.A., I.J. HOOVER and S.G. OGUNGBADE. 1973. The migration and development of Cyathostoma bronchialis in geese. Journal of Parasitology 59: 759-764.

FYVIE, A. and E.M. ADDISON. 1979. Manual of common parasites, diseases, and anomalies of wildlife in Ontario. Ontario Ministry of Natural Resources.

GIBSON, G.G. 1965. The taxonomy and biology of Splendidofilariinae nematodes of the Tetraonidae of British Columbia. Ph.D. thesis, University of British Columbia.

GIBSON, G.G. 1967. Splendidofilaria pectoralis n.sp. (Filarioidea: Onchocercidae) from tetraonid birds in northwestern North America. Canadian Journal of Zoology 45: 1135-1147.

GIBSON, G.G. 1968. Species composition of the genus Streptocara Railliet et al., 1912 and the occurrence of these avian nematodes (Acuariidae) on the Canadian Pacific coast. Canadian Journal of Zoology 46: 629-645.

GIBSON, G.G. 1972. Sciadiocara denticulata n.sp. (Acuariidae) from Actitis macularia (L.) and other nematodes from spotted sandpiper and black-bellied plover. Canadian Journal of Zoology 50: 131-136.

GIBSON, G.G. 1973. Cardiofilaria pavlovskyi Strom, 1937 and Avioserpens sp. (Nematoda) from Canadian ciconiiform birds. Canadian Journal of Zoology 51: 847-851.

GLOVER, J.S. 1947. A brief review of some of the causes of morbidity and mortality in Ontario poultry flocks during the past fiscal year. Report of the Ontario Veterinary College for 1946, pp. 63-67.

GODFREY, W.E. 1986. The birds of Canada. Friesen and Sons Ltd, Ottawa.

GRIFFITHS, H.J. 1947. A record of Trichostrongylus tenuis from domestic goose in Canada. Journal of Parasitology 33: 282.

HEMSLEY, R.V. 1971. Fourth-stage Ascaridia spp. larvae associated with high mortality in turkeys. Canadian Veterinary Journal 12: 147-149.
HODASI, J.K.M. 1963. Helminths from Manitoba birds. Canadian Journal of Zoology 41: 1227-1231.

HODASI, J.K.M. 1966. A note on some helminths of Manitoba chickens. Canadian Journal of Comparative Medicine 30: 26-27.

HOOD, D.E. and H.E. WELCH 1980. A seasonal study of the red-winged blackbird (Agelaius phoeniceus L.) in Manitoba and Arkansas. Canadian Journal of Zoology 58: 528-537.

HOWARD, R. and A. MOORE. 1984. A complete checklist of the birds of the world. Macmillan Publ. Ltd., London.

IRWIN, J.C. 1975. Mortality factors in whistling swans at Lake St. Clair, Ontario. Journal of Wildlife Diseases 11: 8-12.

JEWER, D.D. and W. THRELFALL. 1978. Parasites of the Fox Sparrow (Passerella iliaca) and Northern Waterthrush (Seiurus noveboracensis) in Newfoundland, Canada. Proceedings of the Helminthological Society of Washington 45: 270-272.

JUDD, W.W. 1959. Nematode worms, Diplotriaena tricuspis (Fedt.) from the body cavity of a Nashville warbler at London, Ontario. Canadian Field Naturalist 73: 130.

KARSTAD, L. and L. SILEO. 1971. Causes of death in captive wild waterfowl in the Kortwright Waterfowl Park, 1967-1970. Journal of Wildlife Diseases 7: 236-241.

KINGSCOTE, A.A. 1957. Fish, wildlife, and zoo animals. Report of the Ontario Veterinary College for 1956 pp. 26-27.

MACKENZIE, D.I., C.E. MCKENZIE and L.W. BROWNLIE. 1979. Comparison of the helminth fauna of eastern and western kingbirds at Delta Marsh, Manitoba. Canadian Journal of Zoology 57: 1143-1149.

MACNEILL, A.C. 1970. Amidostomum anseris infection in wild swans and goldeneye ducks. Canadian Veterinary Journal 11: 164-167.

MACNEILL, A.C. 1975. Case report. Heartworm, Sarconema sp. infection in a whistling swan, Olor columbianus. Canadian Veterinary Journal 16: 82-83. 
MACNEILL, A.C. and T. BARNARD. 1978.

Necropsy results in free-living and captive Anatidae in British Columbia. Canadian Veterinary Journal 19: 17-21.

MAHONEY, S.P. and W. THRELFALL. 1978. Digenea, Nematoda and Acanthocephala of two species of ducks from Ontario and eastern Canada. Canadian Journal of Zoology 56: 436-439.

MAWSON, P.M. 1956a. Ascaroid nematodes from Canadian birds. Canadian Journal of Zoology 34: 35-47.

MAWSON, P.M. 1956b. Capillarid worms from Canadian birds. Canadian Journal of Zoology 34: 163-164.

MAWSON, P.M. 1956c. Trichostrongylid worms from Canadian birds. Canadian Journal of Zoology 34: 164-165.

MAWSON, P.M. 1956d. Three new species of spirurid nematodes from Canadian birds. Canadian Journal of Zoology 34: 193-199.

MAWSON, P.M. 1956e. Spirurid nematodes from Canadian birds. Canadian Journal of Zoology 34: 206.

MAWSON, P.M. 1957. Filariid nematodes from Canadian birds. Canadian Journal of Zoology 35: 213-219.

MCCRAW, B.M. 1952. Gizzard worm (Amidostomum) in geese. Canadian Journal of Comparative Medicine 16: 342.

MCKELVEY, R.W. and A.C. MACNEILL. 1981. Mortality factors of wild swans in British Columbia, Canada. In: Proceedings of the Second International Swan Symposium, Sapporo, Japan. C.V.T. Matthew and M. Smart (editors). International Waterfowl Research Bureau, Slimbridge Glasgow, U.K. pp. 312-318.

MCLAUGHLIN, J.D. and M.D.B. BURT. 1979. A survey of the intestinal helminths of waterfowl from New Brunswick, Canada. Canadian Journal of Zoology 57: 801-807.

MCNEILL, M.A. 1988. Development, pathogenicity and epizootiology of Porrocaecum ensicaudatum (Nematoda: Ascaridoidea) in birds. Ph.D. Thesis, University of Guelph, Guelph, Ontario.

MEASURES, L.N. 1987. Epizootiology, transmission and pathogenicity of Eustrongyloides tubifex (Dioctophymatoidea) in piscivorous birds. Ph.D. Thesis, University of Guelph, Guelph, Ontario.

MITCHELL, W.R. 1953. Observations on poultry diseases in Ontario. Report of the Ontario Veterinary College for 1952 pp. 78-79.

MORGAN, B.B. and E. SCHILLER. 1950. A note on Porrocaecum depressum (Zeder, 1800) (Nematoda: Anisakinae). Transactions of the American Microscopical Society 69: 210-213.

NEUFELD, J.L. 1987. Cross-Canada disease report. Manitoba. Ascaridiasis in turkeys. Canadian Veterinary Journal 28: 108.

NOSEWORTHY, S.M. and W. THRELFALL. 1978. Some metazoan parasites of ring-necked ducks, Aythya collaris (Donovan), from Canada. Journal of Parasitology 64: 365-367.

OLIVER, W.T. 1952. Amidostomiasis in domestic geese. Canadian Journal of Comparative Medicine 16: 235-237.

OLSEN, O.W. and C.E. BRAUN. 1971. Diplotriaena lagopus and D. andersoni spp. $\mathrm{n}$. (Diplotriaenidae: Filarioidea) from white-tailed ptarmigan (Lagopus leucurus) in North America. Proceedings of the Helminthological Society of Washington 38: 86-89.

RAMALINGAM, S. and W.M. SAMUEL. 1978. Helminths in the great horned owl, Bubo virginianus, and snowy owl, Nyctea scandiae, of Alberta. Canadian Journal of Zoology 56: 2454-2456.

RAO, N.S.K. 1951. Paracuaria macdonaldi n.g., n.sp. (Family Acuariidae, subfamily Acuariinae) from the sea gull (Larus argentatus). Canadian Journal of Zoology 29: 167-172.

RAU, M.E., M.S. SHAAR and R. MARKELL. 1978. Les parasites des animaux sauvages du Quebec. 4. Les helminthes du carouge a epaulettes (Agelaius phoeniceus) de la region sud-ouest du Quebec. Naturaliste Canadien 105: 58-59. 
RAYNER, J.A. 1932. Parasites of wild birds in Quebec. Scientific Agriculture 12: 307-309.

RIDDELL, C. and A. GAJADHAR. 1988. Cecal and hepatic granulomas in chickens associated with Heterakis gallinarum infection. Avian Diseases 32: 836-838.

SCOTT, M.E. 1984. Helminth community in the belted kingfisher, Ceryle alcyon (L.), in southern Quebec. Canadian Journal of Zoology 62: 2679-2681.

SHILLINGER, J.E. 1936. Parasites in wildlife. In: Report of committee on parasitic diseases. W. Giltner (ed.). Journal of American Veterinary Medical Association 41: 423-431.

\section{SILEO, L., L. KARSTAD, R. FRANK, M.V.H.} HOLDRINET, E. ADDISON and H.E. BRAUN. 1977. Organochlorine poisoning of ring-billed gulls in southern Ontario. Journal of Wildlife Diseases 13: 313-322.

SPECKMAN, G. 1976. Necropsy findings in various avian species other than domestic chickens and turkeys. Journal of Zoo Animal Medicine 7: 27-30.

STOCK, T.M. and J.C. HOLMES. 1987. Host specificity and exchange of intestinal helminths among four species of grebes (Podicepdidae). Canadian Journal of Zoology 65: 669-676.

STOCK, T.M. and J.C. HOLMES. 1988. Functional relationships and microhabitat distributions of enteric helminths of grebes (Podicipedidae): the evidence for interactive communities. Journal of Parasitology 74: 214-227.

SWALES, W.E. 1933a. Tetrameres crami sp.nov., a nematode parasitizing the proventriculus of a domestic duck in Canada. Canadian Journal of Research 8: 334-336.

SWALES, W.E. 1933b. A review of Canadian helminthology: I. The present status of knowledge of the helminth parasites of domesticated and semi-domesticated mammals and economically important birds in Canada, as determined from work published prior to 1933. Canadian Journal of Research 8: 468-477.
SWALES, W.E. 1936. Two important diseases in ducks in Quebec. Journal of Agriculture and Horticulture Quebec 39: 13, 40-43.

THRELFALL, W. 1968a. The helminth parasites of three species of gulls in Newfoundland. Canadian Journal of Zoology 46: 827-830.

THRELFALL, W. 1968b. Helminth parasites of some birds in Newfoundland. Canadian Journal of Zoology 46: 909-913.

THRELFALL, W. 1968c. Studies on the helminth parasites of the American herring gull (Larus argentatus Pont.) in Newfoundland. Canadian Journal of Zoology 46: 1119-1126.

THRELFALL, W. 1968d. Helminths recovered from Wilson's snipe, Capella gallinago delicata. Journal of Helminthology 42: 173-178.

THRELFALL, W. 1971. Helminth parasites of alcids in the northwestern Atlantic. Canadian Journal of Zoology 49: 461-466.

THRELFALL, W. 1981. Helminth parasites of an American flamingo from Newfoundland, Canada. Proceedings of the Helminthological Society of Washington 48: 89-90.

TUGGLE, B.M. and J.L. CRITES. 1984. The prevalence and pathogenicity of gizzard nematodes of the genera Amidostomum and Epomidiostomum (Trichostrongylidae) in the lesser snow goose (Chen caerulescens caerulescens). Canadian Journal of Zoology 62: 1849-1852.

TURNER, B.C. and W. THRELFALL. 1975. The metazoan parasites of green-winged teal (Anas crecca L.) and blue-winged teal (Anas discors L.) from eastern Canada. Proceedings of the Helminthological Society of Washington 42: 157-169.

VANDERBURGH, D.J., R.C. ANDERSON and T.M. STOCK. 1984. Pelecitus tubercauda n.sp. (Nematoda: Filarioidea) from Geothlypis trichas $\mathrm{L}$. and a redescription of $P$. fulicaeatrae (Diesing, 1861) Lopez-Neyra, 1956. Canadian Journal of Zoology 62: 362-367.

VERMEER, K. 1969. Comparison of the helminth fauna of California gulls, Larus californicus, and ring-billed gulls, Larus delawarensis at Beaverhill and Miquelon Lakes, Alberta. Canadian Journal of Zoology 47: 267-270. 
WEBSTER, W.A. 1974. Diplotriaena obtusa (Rud., 1802) and Plagiorchis maculosus (Rud., 1802) collected from a purple martin, Progne subis, in Canada. Proceedings of the Helminthological Society of Washington 41: 109.

WEBSTER, W.A. and G. SPECKMAN. 1976. Diplotriaena darnaudii sp.n. (Nematoda: Diplotriaenidae) from D'Arnaud's barbet, Trachyphonus darnaudii. Journal of Parasitology 62: 451-452.

WICKWARE, A.B. 1940. Lead poisoning in wild ducks following ingestion of shot. Canadian Journal of Comparative Medicine 4: 201-203.

WICKWARE, A.B. 1941. Notes on miscellaneous diseases of geese. Canadian Journal of Comparative Medicine 5: 21-24.

WOBESER, G.A. 1981. Diseases of wild waterfowl. Plenum Press, New York.

WONG, P.L. 1985. Revision of the genus Stellocaronema Gilbert, 1930 (Nematoda: Habronematoidea). Canadian Journal of Zoology 63: 2430-2436.

WONG, P.L. and R.C. ANDERSON. 1982a. Redescription of Paracuaria adunca (Creplin, 1846) (Nematoda: Acuarioidea) from Larus delawarensis Ord (Laridae). Canadian Journal of Zoology 60: 175-179.

WONG, P.L. and R.C. ANDERSON. $1982 \mathrm{~b}$. Redescription of Pectinospirura argentata Wehr, 1933 (Nematoda: Acuarioidea) from Larus delawarensis Ord (Laridae). Canadian Journal of Zoology 60: 1940-1944.

WONG, P.L. and R.C. ANDERSON. 1983. Revision of the genera Cordonema Schmidt and Kuntz, 1972 and Skrjabinoclava Sobolev, 1943 (Nematoda: Acuarioidea). Canadian Journal of Zoology 61: 339-348.

WONG, P.L. and R.C. ANDERSON. 1990. Ancyracanthopsis winegardi n.sp. (Nematoda: Acuarioidea) from Pluvialis squatarola (Aves: Charadriidae) and Ancyracanthopsis heardi n.sp. from Rallus longirostris (Aves: Rallidae), and a review of the genus. Canadian Journal of Zoology 68: 1297-1306.

WONG, P.L. and R.C. ANDERSON. In press. Host and geographic distribution of Skrjabinoclava spp. (Nematoda: Acuarioidea) in nearctic shorebirds (Aves: Charadriiformes) and evidence for transmission in marine habitats in staging and wintering areas. Canadian Journal of Zoology.

WONG, P.L., R.C. ANDERSON, R.C. and C.M. BARTLETT. 1986. Revsion of the genus Syncuaria Gilbert, 1927 (Nematoda: Acuarioidea). Canadian Journal of Zoology 64: 1186-1196.

WONG, P.L., R.C. ANDERSON and J. FRIMETH. 1983. Diplotriaena utae sp.n. (Nematoda: Diplotriaenoidea) in the gray jay (Perisoreus canadensis (L.)) in Ontario, Canada. Proceedings of the Helminthological Society of Washington 50: 275-277.

WONG, P.L., A.O. BUSH and R.C. ANDERSON. 1987. Redescription of Skrjabinocerca prima Shikhobalova, 1930 (Nematoda: Acuarioidea) from the American avocet (Recurvirostra americana Gmelin). Canadian Journal of Zoology 65: 1569-1573.

WONG, P.L. and M.W. LANKESTER. 1984a. Revision of the genus Schistorophus Railliet, 1916 (Nematoda: Acuarioidea). Canadian Journal of Zoology 62: 2527-2540.

WONG, P.L. and M.W. LANKESTER. 1984b. Revision of the genus Viktorocara Guschanskaya, 1950 (Nematoda: Acuarioidea). Canadian Journal of Zoology 62: 2541-2549.

WONG, P.L. and M.W. LANKESTER. 1985a. Revision of the genus Ancyracanthopsis Diesing, 1861 and description of a new genus Molinacuaria n.gen. (Nematoda: Acuarioidea). Canadian Journal of Zoology 63: 1556-1564.

WONG, P.L. and M.W. LANKESTER. 1985b. Revision of the genus Sciadiocara Skrjabin, 1916 (Nematoda: Acuarioidea). Canadian Journal of Zoology 63: 1565-1575.

WONG, P.L. and M.W. LANKESTER. 1985c. Revision of the genus Sobolevicephalus Parukhin, 1964 (Nematoda: Acuarioidea). Canadian Journal of Zoology 63: 1575-1581.

WRIGHT, R.R. 1879. Contributions to American helminthology. No. 1. Proceeding of the Canadian Institute 1: 54-75. 
Acanthophorus horridus ....... 8

Acanthophorus tenuis ......... 8

Acuaria (Dispharynx) nasuta . . . . . . . 10

Acuaria (Hamannia) uncinata . . . . . . 10

Acuaria anthuris ............ 9

Acuaria ornata ........... . . 9

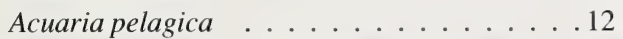

Acuaria species ............. 9

Acuaria spinosa ............ . . . . 9

Acuaria spiralis .......... . . . . . 10

Acuaria squamata . . . . . . . . . . . 12

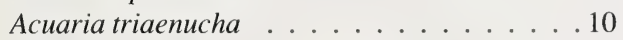

ACUARIIDAE .............. 9

AMIDOSTOMATIDAE ......... 4

Amidostomum acutum ........... . 4

Amidostomum anseris .......... 5

Amidostomum cygni ............ 5

Amidostomum spatulatum ......... 5

Amidostomum species .......... . . 5

Ancyracanthopsis bendelli ........ . . 12

Ancyracanthopsis bihamata ....... . . 14

Ancyracanthopsis bilabiatus ....... 13

Ancyracanthopsis coronata ..... 13, 14

Ancyracanthopsis heardi ....... . . . 13

Ancyracanthopsis winegardi . . . . . . . 14

Ancyracanthus bihamata ........ . . 14

Ancyracanthus bilabiatus ......... 13

ANISAKIDAE ............... 6

Antennocara skrjabini ............ 14

Aprocta turgida . . . . . . . . . . 16

Aproctella species . . . . . . . . . . . . . 17

Aproctella stoddardi . . . . . . . . . . 17

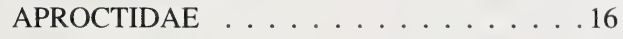

Ascaridia bonasae ......... 6

Ascaridia compar ........... 6

Ascaridia galli ............ . . . . . . . . . . . . 6

Ascaridia lineata . . . . . . . . . . 6

Ascaridia species fourth-stage larvae . . . . 6

ASCARIDIDAE ........... 7

Ascarididae, unidentified larvae ... . . 7

ASCARIDIIDAE ........... 6

Aviculariella alcyona . . . . . . . . . . . . . . . . .

Avioserpens nana . . . . . . . . . . . 7

Avioserpens species ........... 7

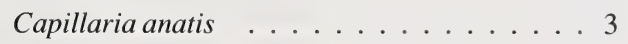

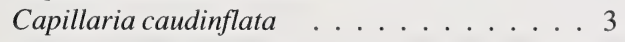

Capillaria columbae .......... 3

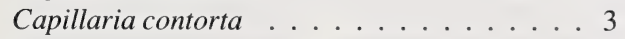

Capillaria falconis-nisi ......... 3

Capillaria mergi ................. 3

Capillaria nyrocinarum ......... 3

Capillaria obsignata .......... 3

Capillaria ovopunctatum ......... 3
Capillaria quiscala ......................

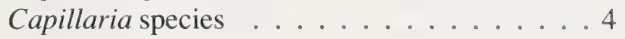

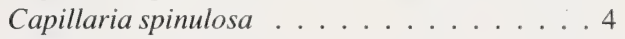

Capillaria tridens .............. 4

Cardiofilaria pavlovskyi .......... 17

Carinema ardea ............ 17

Chandlerella bushi ........... 18

Chandlerella chitwoodae ......... 18

Chandlerella hispanica ......... 18

Chandlerella quiscali ............ 18

Cheilospirura capillaris ......... 14

Cheilospirura longistriata ......... . 8

Cheilospirura nasuta .......... 10

Cheilospirura spinosa .......... 9

Chevreuxia americana .......... 10

Chordocephalus squamata . . . . . 12

Contracaecum anasi ... . . . . . . . . 6

Contracaecum magnicollare ....... . 6

Contracaecum microcephalum ... . . . 6

Contracaecum ovale . . . . . . . . . . 6

Contracaecum pelagicum ......... 6

Contracaecum podicipitis ......... 6

Contracaecum species .......... . . 7

Contracaecum spiculigerum . . . . . . . . . 6

Contracaecum yamaguti . . . . . . . . . . . 6

Cosmocephalus aduncus ......... . . . 10

Cosmocephalus capellae ........ 10

Cosmocephalus diesingi . . . . . . . . . . 10

Cosmocephalus faridi . . . . . . . . . 10

Cosmocephalus firlottei . . . . . . . . 10

Cosmocephalus obvelatus . . . . . . . . 10

Cosmocephalus obvelatus magnus ... . 10

Cosmocephalus tanakai . . . . . . . . . 10

Cyathostoma bronchialis ......... 4

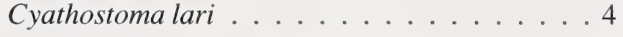

Cyathostoma species .......... 4

Cyrnea americanum .......... 8

Cyrnea longispicula ............ 8

Desmidocerca incognita . . . . . . . . 16

Desmidocercella nudicauda . . . . . . . 16

Desmidocercella numidica ......... 16

DESMIDOCERCIDAE ......... 16

Desportesius canadensis . . . . . . . . . . 10

Desportesius triaenucha . . . . . . . . . . . 10

Dicheilonematid immature worms . . . . 15

DIOCTOPHYMATIDAE ............. 3

Diplotriaena agelaius . . . . . . . . . . 15

Diplotriaena andersoni .......... 15

Diplotriaena bargusinica . . . . . . . 15

Diplotriaena darnaudii . . . . . . . . 15

Diplotriaena isabellina . . . . . . . . 15

Diplotriaena obtusa .......... 15

Diplotriaena ozouxi .......... 15 
Diplotriaena pungens . . . . . . . . . 15

Diplotriaena sialiae . . . . . . . . . . 15

Diplotriaena species . . . . . . . . . 16

Diplotriaena sylvinae . . . . . . . . . . . 15

Diplotriaena thomasi . . . . . . . . . . 15

Diplotriaena tricuspis . . . . . . . . . . 15

Diplotriaena utae . . . . . . . . . . . . 16

DIPLOTRIAENIDAE . . . . . . . . . 14

Diplotriaenoides translucidus . . . . . . . 15

Dispharagus aduncus . . . . . . . . . . .11

Dispharagus anthuris . . . . . . . . . 99

Dispharagus columbae . . . . . . . . . 10

Dispharagus crassicauda . . . . . . . . . 13

Dispharagus nasutus . . . . . . . . . . 10

Dispharagus nasutus . . . . . . . . . . 10

Dispharagus obvelatus . . . . . . . . . 10

Dispharagus spiralis . . . . . . . . . . 10

Dispharagus squamatus . . . . . . . . . . 12

Dispharagus uncinata . . . . . . . . . . . 10

Dispharynx nasuta . . . . . . . . . 10

DRACUNCULIDAE .......... . . 7

Echinuria borealis . . . . . . . . 10

Echinuria hypognatha . . . . . . . 10

Echinuria jugadornata ......... . 10

Echinuria parva . . . . . . . . . 10

Echinuria spinosa . . . . . . . . . . 9 9

Echinuria squamata . . . . . . . . . . 12

Echinuria uncinata . . . . . . . . . 10

Epomidiostomum crami . . . . . . . . . 5 5

Epomidiostomum uncinatum . . . . . . . 5 5

Eufilaria longicaudata . . . . . . . 16

Eufilaria mcintoshi ........... . . 16

Eufilaria sturninus . . . . . . . . 16 16

Eulimdana asperum . . . . . . . . . . . 16

Eulimdana florencae . . . . . . . . 17

Eulimdana juventarum . . . . . . . . 17

Eulimdana pseudolari . . . . . . . . . . 17

Eulimdana species . . . . . . . . . 17

Eustrongylides elegans . . . . . . . . 3

Eustrongylides mergorum . . . . . . . . . . 3

Eustrongylides perpapillatus . . . . . . . 3

Eustrongylides tubifex . . . . . . . . 3

Filaria anthuris . . . . . . . . . 9

Filaria obvelatus . . . . . . . . 10

Filaria pulicis . . . . . . . . . 8

Filaria recta . . . . . . . . . . . 13

Filaria squamata . . . . . . . . . 12

Filaria triaenucha ............. . 10

Filaria tridentata ............ 11

Geopetitia aspiculata . . . . . . . . . 9 9

Gnathostoma shipleyi . . . . . . . . . . 12

Habronema mansioni . . . . . . . . . . 8

HABRONEMATIDAE .......... 8

Hamannia squamata . . . . . . . . . 12

Hamannia uncinata . . . . . . . . . 10
Hamatospiculum species . . . . . . . . . . 14

HETERAKIDAE ........... . . 6

Heterakis gallinae . . . . . . . . . . 6

Heterakis gallinarum . . . . . . . . 6

Heterakis isolonche . . . . . . . . . . 6

Histiocephalus coronata . . . . . . . . 13

Histiocephalus stellae-pollaris . . . . . . 12

Lemdana wernaarti . . . . . . . . . . 17

Microtetrameres bubo . . . . . . . . . 8

Microtetrameres canadensis . . . . . . . .8

Microtetrameres helix . . . . . . . . . 8 8

Microtetrameres species . . . . . . . . . 8

Molinacuaria bendelli . . . . . . . . . . . 12

Monopetalonema alcedinis . . . . . . . . 15

ONCHOCERCIDAE .......... 16

Ornithofilaria algonquinensis . . . . . . . . 18

Ornithofilaria fallisensis . . . . . . . . . . . 18

Ornithofilaria inornata . . . . . . . . 17

ORNITHOSTRONGYLIDAE . . . . . 5

Ornithostrongylus quadriradiatus . . . . . . 5 5

Oxyspirura lumsdeni . . . . . . . . . . . 7

Oxyspirura petrowi .......... . . 7

Paracuaria adunca . . . . . . . . 11

Paracuaria macdonaldi .......... 11

Paracuaria tridentata . . . . . . . . 11

Pectinospirura argentata . . . . . . 11

Pectinospirura sobolevi . . . . . . . . 11

Pelecitus fulicaeatrae . . . . . . . . 16

Pelecitus tubercauda . . . . . . . . . . . 16

Physocephalus sexalatus third-stage larvae . . 7

Porrocaecum brevispiculum . . . . . . . . 7

Porrocaecum depressum . . . . . . . . . . . 7

Porrocaecum ensicaudatum . . . . . . . . 7

Porrocaecum semiteres . . . . . . . . . . 7

Porrocaecum species . . . . . . . . . 7

Porrocaecum trichuriforme . . . . . . . . 7

Prionostemma pelagicum . . . . . . . 12

Prionostemma shipleyi . . . . . . . . . . . 12

Pseudaprocta samueli . . . . . . . . . 16

Quadriplotriana hypsokysta ........ 16

Rictularia paradoxa . . . . . . . . 12

Rictularia shipleyi ... . . . . . . 12

Robertdollfusa species . . . . . . . . . . 4

ROBERTDOLLFUSIDAE . . . . . . . 4

Rusguniella transcaucasica . . . . . . . 11

Sarconema eurycerca . . . . . . . . . 17

Sarconema similar to S. eurycerca . . . . 17

Sarconema species . . . . . . . . . . . 17

Schistorophus bihamatus ........ 14

Schistorophus capillaris . . . . . . . . . 14

Schistorophus skrjabini . . . . . . . . . 14

Schistorophus umbellifera . . . . . . . . 14

Sciadiocara bihamata . . . . . . . . . 14

Sciadiocara denticulata . . . . . . . . . 14 
Sciadiocara umbellifera . . . . . . . . . 14

Serratospiculoides amaculatum . . . . . 15

Serratospiculum amaculata . . . . . . . . 15

Seuratia shipleyi . . . . . . . . . . 12

Skrjabinobronema californica . . . . . . 12

Skrjabinobronema coronata . . . . . . . 13

Skrjabinocara schikhobalovi . . . . . . . 12

Skrjabinocara skrjabini . . . . . . . . . . 12

Skrjabinocara squamata . . . . . . . . 12

Skrjabinocara timofejevi . . . . . . . . . 12

Skrjabinocara viktori . . . . . . . . . . 12

Skrjabinocerca prima . . . . . . . . . 11

Skrjabinoclava bakeri ............11

Skrjabinoclava bartlettae ...........11

Skrjabinoclava horrida . . . . . . . . 11

Skrjabinoclava inornatae . . . . . . . 11

Skrjabinoclava kritscheri . . . . . . . . . 11

Skrjabinoclava morrisoni . . . . . . . . . 11

Skrjabinoclava pusillae . . . . . . . . . 12

Skrjabinoclava tupacincai ... . . . . . 12

Sobolevicephalus lichtenfelsi . . . . . . . . 14

SPIROCERCIDAE ............ . . 7

Spiroptera adunca ............ . 11

Spiroptera adunca ............ 10

Spiroptera anthuris ........... . . 9

Spiroptera capillaris .......... . . 14

Spiroptera coronata . . . . . . . . . 13

Spiroptera crassicauda ... . . . . . 13

Spiroptera inflata . . . . . . . . . 3

Spiroptera longistriata ......... 8

Spiroptera nasuta . . . . . . . . . 10

Spiroptera obvelatus ............ 10

Spiroptera recta ............... . . 13

Spiroptera tantali rubri ... . . . . . . . 14

Spiroptera totani . . . . . . . . . . 14

Spiroptera tridentata . . . . . . . . 11

Spiroptera umbellifera . . . . . . . . 14

Spiroptera uncinata . . . . . . . . . . 10

Splendidofilaria algonquinensis . . . . . 18

Splendidofilaria caperata . . . . . . . . . 18

Splendidofilaria fallisensis . . . . . . . . 18

Splendidofilaria flexivaginalis . . . . . . 18

Splendidofilaria passerina . . . . . . 18

Splendidofilaria pectoralis . . . . . . . 18

Splendidofilaria species . . . . . . . . . 18

Splendidofilaria wehri . . . . . . . . 18

Stegophorus species . . . . . . . . . . . 12

Stegophorus stellae-pollaris _. . . . . . 12

Stellocaronema glareolae . . . . . . . . . 8

Stellocaronema skrjabini . . . . . . . . . 8 8

Streptocara c. crassicauda . . . . . . . 13

Streptocara californica . . . . . . . . . 12

Streptocara crassicauda . . . . . . . . 13

Streptocara crassicauda anseri . . . . . . . . 13

Streptocara formosensis . . . . . . . . 13

Streptocara incognita . . . . . . . . . . 13

Streptocara recta .............. . 13
Streptocara rissae ............ 11

Streptocara species ............ 13

Streptocara stellae-pollaris . . . . . . . 12

Streptocara transcaucasica . . . . . . . 11

Streptocara triaenucha . . . . . . . . 10

Streptocara tridentata . . . . . . . . 13

Strongyloides species . . . . . . . . . 4

STRONGYLOIDIDAE . . . . . . . . . 4

Strongylus tubifex . . . . . . . . . . . . 3

Syncuaria squamata . . . . . . . . . 12

SYNGAMIDAE .............. . 4

Syngamus trachea . . . . . . . . . . . . 4

Synhimantus species . . . . . . . . . 12

Tetrameres ardamericanus . . . . . . . 8

Tetrameres crami . . . . . . . . . . 8

Tetrameres fissispina .......... 8

Tetrameres occinea . . . . . . . . . 9 9

Tetrameres ryjikovi . . . . . . . . . .99

Tetrameres somateriae . . . . . . . . 99

Tetrameres species . . . . . . . . . . . 99

Tetrameres spinosa . . . . . . . . . . 9

TETRAMERIDAE .......... 8

THELAZIDAE $\ldots \ldots \ldots \ldots \ldots . \ldots$

TRICHOSTRONGYLIDAE . . . . . . . . 5

Trichostrongylus tenuis . . . . . . . . 5

TRICHURIDAE . . . . . . . . . . 3

Tropidocerca fissispina ........ . . 8

Tropidocerca paradoxa . . . . . . . . 3

Tropisurus fissispina . . . . . . . . 8

Unidentified Skrjabinoclava larvae $\ldots \ldots 12$

Viktorocara capillaris . . . . . . . . 14

Viktorocara halcyoni . . . . . . . . . 14

Viktorocara limosae . . . . . . . . . . 14

Viktorocara numenii . . . . . . . . . . . . 14

Viktorocara schejkini . . . . . . . . . 14

Viktorocara torea ............ . 14

Yseria californica . . . . . . . . 12

Yseria coronata ............. 13

Yseria stellae-pollaris . . . . . . . . 12 


\section{HOST INDEX}

Accipiter gentilis . . . . . . . . . 22

Accipiter striatus . . . . . . . . . . 22

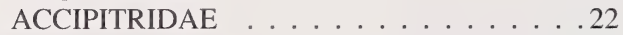

Actitis macularia . . . . . . . . . . . . . 24

Actodromus bairdii . . . . . . . . . . . 24

Aechmophorus occidentalis . . . . . . . . . 19

Agelaius phoeniceus . . . . . . . . . 30

Alca lomvia . . . . . . . . . . 26

Alca torda . . . . . . . . . . . . 26

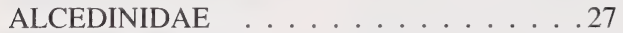

Alcedo alcyon . . . . . . . . . . . . . 27

ALCIDAE . . . . . . . . . . . 26

Anas acuta . . . . . . . . . . . . . . 20

Anas albeola .............. . . 21

Anas caerulescens . . . . . . . . . . . 20

Anas canadensis . . . . . . . . . . . 20

Anas clangula . . . . . . . . . . . 21

Anas clypeata . . . . . . . . . . . 20

Anas collaris .............. . . 21

Anas columbianus . . . . . . . . . . . 20

Anas crecca . . . . . . . . . . . . . 21

Anas discors . . . . . . . . . . . . . . 21

Anas fusca . . . . . . . . . . . . . . 22

Anas hyemalis . . . . . . . . . . . 21

Anas islandica . . . . . . . . . . . . . . 21

Anas jamaicensis . . . . . . . . . . . 22

Anas marila . . . . . . . . . . . . . 21

Anas mollissima . . . . . . . . . . . . 22

Anas nigra . . . . . . . . . . . . . . . . 22

Anas perspicillata . . . . . . . . . . . 22

Anas platyrhynchos (domestic) . . . . . . .21

Anas platyrhynchos (mallard) . . . . . . . 21

Anas rubripes . . . . . . . . . . . . 21

Anas spectabilis . . . . . . . . . . . . . 22

Anas strepera . . . . . . . . . . . . 21

Anas valisineria . . . . . . . . . . . . . 21

ANATIDAE ................. 20

Anitibyx armatus . . . . . . . . . . . . . . . . . . . . . . . . . . .

Anser anser . . . . . . . . . . . . . 20

Anser hyperboreus . . . . . . . . 20

Ardea exilis . . . . . . . . . . . . 20

Ardea herodias . . . . . . . . . . . . . 19

Ardea lentiginosa . . . . . . . . . . 20

ARDEIDAE . . . . . . . . . . . . . . . 19

Asioflammeus . . . . . . . . . . . . . 27

Asio otus . . . . . . . . . . . . . . . 27

Aythya americana . . . . . . . . . . 21

Aythya collaris .............21

Aythya marila . . . . . . . . . . . . 21

Aythya valisineria . . . . . . . . . . . . 21

Bartramia longicauda . . . . . . . . . . . 24

Bombycilla cedrorum . . . . . . . . . . . 29

BOMBYCILLIDAE . . . . . . . . . . 29
Bonasa umbellus . . . . . . . . . . . . 23

Botaurus lentiginosus . . . . . . . . 20

Branta canadensis . . . . . . . . . . 20

Branta species ... . . . . . . . . 20

Bubo virginianus . . . . . . . . . . . . . 27

Bucephala albeola . . . . . . . . . 21

Bucephala clangula ... . . . . . 21

Bucephala islandica . . . . . . . . . 21

Buteo jamaicensis ... . . . . . . . 23

Calidris alba . . . . . . . . . . . . . 24

Calidris alpina . . . . . . . . . . . . 24

Calidris bairdii . . . . . . . . . . . . . . 24

Calidris himantopus . . . . . . . . . . . 24

Calidris mauri . . . . . . . . . . . 25

Calidris minutilla . . . . . . . . . . 25

Calidris pusilla . . . . . . . . . . . 25

Callipepla californica . . . . . . . 23

Capella gallinago . . . . . . . . . 25

CAPITONIDAE ............. . . 27

Carbo auritus . . . . . . . . . . . 19

Catharus fuscescens . . . . . . . . . . . 28

Catharus ustulatus . . . . . . . . . . . 28

Catoptrophorus semipalmatus inornatus . . 25

Centrocercus urophasianus . . . . . . . . 23

Cepphus columba . . . . . . . . . 26

Ceryle alcyon . . . . . . . . . . 27

CHARADRIIDAE ............. . . 24

Charadrius semipalmatus . . . . . . . . . 24

Charadrius vociferus . . . . . . . . . . . 24

Chen caerulescens . . . . . . . . . 20 20

Chen caerulescens atlanticus . . . . . . . 20

Chlidonias niger . . . . . . . . . . . . . 26

Chloropsis hardwikei . . . . . . . . . . 28

Circus cyaneus . . . . . . . . . . . . 23

Clangula hyemalis . . . . . . . . . . 21

Columba domestica livia . . . . . . . . 26 26

Columba livia . . . . . . . . . . . . 26

Columba species ... . . . . . . . 26 26

COLUMBIDAE ............... 26

Colymbus aalge ............. . . 26

Colymbus auritus . . . . . . . . . . . . 19

Colymbus grisegena . . . . . . . . . . 19

Colymbus immer . . . . . . . . . . . . . . . . 19

Colymbus podiceps . . . . . . . . . 19

Colymbus stellatus . . . . . . . . . . . 19

Coracias galbula . . . . . . . . . . . . 30 30

CORVIDAE . . . . . . . . . . . . . 28

Corvus brachyrhynchos . . . . . . . . . 28

Corvus canadensis . . . . . . . . . . 28

Corvus corax . . . . . . . . . . . . . 28

Corvus cristatus . . . . . . . . . . . 28

Cyanocitta cristata . . . . . . . . . . 28

Cygnus buccinator . . . . . . . . . . . 20 


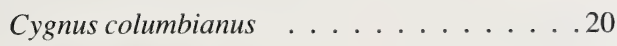

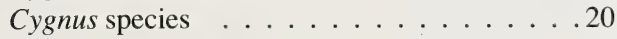

Dendragapus canadensis . . . . . . . . . . . . . . . . . 23

Dendragapus obscurus . . . . . . . . . . . . . . . . . . . . . 23

Dendragapus obscurus fuliginosus . . . . . . . . . . . . . 23

Dendroica coronata . . . . . . . . . . . 29

Dendroica magnolia . . . . . . . . . . . . 29

Dendroica pensylvanica . . . . . . . . . . 29

Diomedea nigripes . . . . . . . . . . . . 19

Diomedea species . . . . . . . . . . . 19

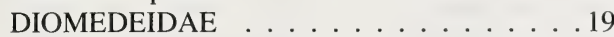

Ducks, geese and swans ........ . . 20

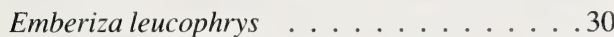

EMBERIZIDAE ................ 29

Ereunetes mauri . . . . . . . . . . . . 25

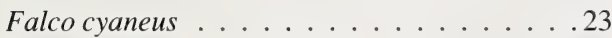

Falco gentilis ........... . . . . 22

Falco jamaicensis . . . . . . . . . . 23

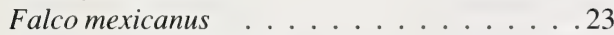

Falco peregrinus anatum . . . . . . . . 23

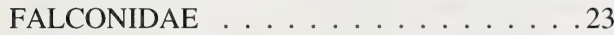

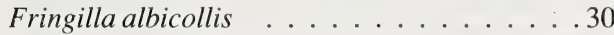

Fringilla erythropthalmus . . . . . . . 29

Fringilla graminea . . . . . . . . . . . 30

Fringilla hyemalis . . . . . . . . . . . . . . . . . . . . . 29

Fringilla iliaca . . . . . . . . . . . . 29

Fringilla melodia . . . . . . . . . . . 29

FRINGILLIDAE . . . . . . . . . . . . . . . . . . . . . . . .

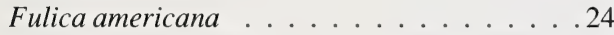

Fuligula americana . . . . . . . . . . 21

Gallinago gallinago ............ 25

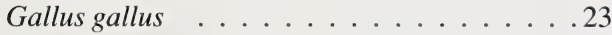

Gavia immer ............ . . 19

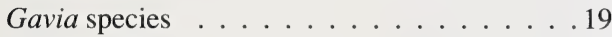

Gavia stellata .............. 19

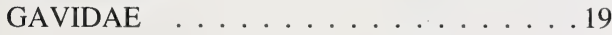

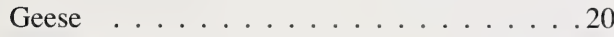

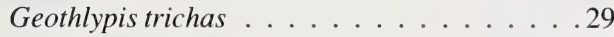

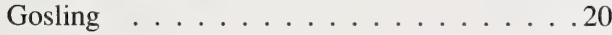

HIRUNDINIDAE . . . . . . . . . . . . . . . . . . . . . . . .

Hirundo pyrrhonota . . . . . . . . . . . . . . . . . . . . .

Hirundo riparia . . . . . . . . . . . . . . . . . . . . . . . . . . . . 28

Hirundo rustica . . . . . . . . . . . 27

Hirundo subis . . . . . . . . . . . . . . . . . . . . . . . . . . . . . .

Hylocichla mustelina . . . . . . . . . 28

Icterus galbula . . . . . . . . . . . . . . . . . . . . . . 30

Icterus xanthocephalus . . . . . . . . . . . . . . . . . . .

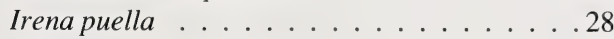

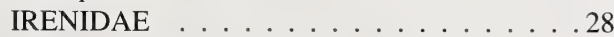

Ixobrychus exilis . . . . . . . . . . 20

Junco hyemalis . . . . . . . . . . . . . 29

Lagopus lagopus . . . . . . . . . . . . . . . . . . . . . . . . 23

Lagopus leucurus . . . . . . . . . . . 23
Lanius jocosus . . . . . . . . . . . . . . . . . . . 28

Lanius sulphuratus . . . . . . . . . . . . 27

Lanius tyrannus . . . . . . . . . . . . . 27

LARIDAE . . . . . . . . . . . . . . 26

Larus argentatus . . . . . . . . . . . . . . 26

Larus californicus . . . . . . . . . . . . . 26

Larus canus . . . . . . . . . . . . . 26

Larus delawarensis . . . . . . . . . . . . 26

Larus glaucescens . . . . . . . . . . . . 26

Larus marinus . . . . . . . . . . . . . . . 26

Larus pipixcan . . . . . . . . . . . 26

Larus thayeri . . . . . . . . . . . . . . . . 26

Larus tridactyla . . . . . . . . . . . . . . 26

Limnodromus scolopaceus . . . . . . . . . 25

Limosa fedoa . . . . . . . . . . . . . . 25

Limosa scolopacea . . . . . . . . . . . . . 25

Lophodytes cucullatus . . . . . . . . . . 22

Lophortyx californica . . . . . . . . . . 23

Loxia oryzivora . . . . . . . . . . . . 30

Melanitta fusca ............ . . 22

Melanitta fusca deglandi . . . . . . . . . 22

Melanitta nigra ............ 22

Melanitta perspicillata .......... 22

Meleagris gallopavo ........... . . . 23

Melospiza melodia . . . . . . . . . . . 29

Mergansers ................ 22

Mergus cucullatus . . . . . . . . . . . 22

Mergus merganser . . . . . . . . . . . 22

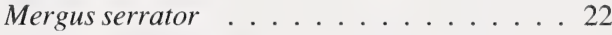

Minla cyanouroptera ........... 28

Molothrus ater ... . . . . . . . . . 30

Motacilla aurocapilla . . . . . . . . . . . . 29

Motacilla coronata . . . . . . . . . . 29

Motacilla currucoides . . . . . . . . . . . 28

Motacilla noveboracensis . . . . . . . . 29

Motacilla pensylvanica . . . . . . . . . . . 29

Muscicapa canadensis . . . . . . . . . . . 29

MUSCICAPIDAE ............ . . . . . . . . . . . . . 28

Myiophoneus caeruleus . . . . . . . . 28 28

Numenius americanus . . . . . . . . . . . 25

Nyctea scandiaca . . . . . . . . . 27

Oriolus ater . . . . . . . . . . . . . . 30

Oriolus phoeniceus ............. 30

Owl .................. 27

Oxyura jamaicensis ........ . . . 22

Padda oryzivora .............. 30

Passerella iliaca . . . . . . . . . . . . . . 29 29

Pedioecetes phasianellus ........ 23

Perisoreus canadensis . . . . . . . . . . . . . . . 28

PHALACROCORACIDAE . . . . . . . . . 19

Phalacrocorax auritus .......... . . 19

Phalaropus lobatus ......... . . 25

Phalaropus tricolor . . . . . . . . . . 25

PHASIANIDAE ............ . . . 23

Phasianus colchicus . . . . . . . . . 23 
PHOENICOPTERIDAE .......... 20

Phoenicopterus ruber . . . . . . . . . . . . . 20

PHOENICULIDAE . . . . . . . . . . 27

Phoeniculus purpureus . . . . . . . . . . .27

Pipilo erythropthalmus . . . . . . . . . . .29

Pitangus sulphuratus . . . . . . . . . . . 27

Pluvialis squatarola . . . . . . . . . . . . . . 24

Podiceps auritus . . . . . . . . . . . . . 19

Podiceps grisegena . . . . . . . . . . . . . 19

Podiceps nigricollis . . . . . . . . . . . . . . 19

Podiceps occidentalis . . . . . . . . . . . . 19

PODICIPEDIDAE ............ . . 19

Podilymbus podiceps . . . . . . . . . . . 19

Pooecetes gramineus . . . . . . . . . . . 29

Procellaria gravis . . . . . . . . . . . . . 19

PROCELLARIIDAE . . . . . . . . . 19

Progne subis . . . . . . . . . . . . . 27

Puffinus gravis . . . . . . . . . . . . 19

PYCNONOTIDAE ............. . . 28

Pycnonotus cafer . . . . . . . . . . . . . 28

Pycnonotus jocosus . . . . . . . . . . . . 28

Quiscalus quiscula . . . . . . . . . . .30

Quiscalus quiscula versicolor . . . . . . 30

RALLIDAE . . . . . . . . . . . . . 24

Recurvirostra americana . . . . . . . 24

RECURVIROSTRIDAE . . . . . . . . . . 24

Riparia riparia . . . . . . . . . . . 28

Rissa tridactyla . . . . . . . . . . 26

Saltator coerulescens . . . . . . . . . 29

SCOLOPACIDAE .............. 24

Scolopax fedoa . . . . . . . . . . . 25

Scolopax flavipes . . . . . . . . . . 25

Scolopax gallinago ........... . . 25

Scolopax melanoleuca ...........25

Scolopax minor . . . . . . . . . . . . 25

Seiurus aurocapillus . . . . . . . . . . 29

Seiurus noveboracensis . . . . . . . . . . 29

Sialia currucoides . . . . . . . . . . 28

Somateria mollissima . . . . . . . . . 22

Somateria spectabilis . . . . . . . . . . 22

Spatula clypeata . . . . . . . . . 20

Steganopus tricolor . . . . . . . . . . . . . 25

Sterna hirundo . . . . . . . . . . . 26

Sterna niger . . . . . . . . . . . 26

STRIGIDAE . . . . . . . . . . . 27

Strix flammea . . . . . . . . . . . . . . 27

Strix otus . . . . . . . . . . . 27

Strix scandiaca . . . . . . . . . . . . . 27

Strix virginianus . . . . . . . . . . . . . . . 27

Sturnella neglecta ............. . . 30

STURNIDAE . . . . . . . . . . . . . . . 29

Sturnus vulgaris . . . . . . . . . . . . . . . 29

Sylvia magnolia . . . . . . . . . . . . . . . 29

Sylvia ruficapilla . . . . . . . . . . . . . . 29

Tachyphonus rufus . . . . . . . . . . . 29
Tanagra episcopus . . . . . . . . . . . . 29

Tanagra palmarum . . . . . . . . . 29

Tangara rufa . . . . . . . . . . . 29

Tetrao californica . . . . . . . . . 23

Tetrao canadensis . . . . . . . . . 23

Tetrao cupido . . . . . . . . . . 23

Tetrao lagopus .............. . . 23

Tetrao leucurus ............. . 23

Tetrao obscurus ............ . 23

Tetrao phasianellus .......... 23

Tetrao umbellus . . . . . . . . . . 23

Tetrao urophasianus . . . . . . . . . 23

Thraupis episcopus . . . . . . . . . . 29

Thraupis palmarum . . . . . . . . . . 29

Trachyphorus darnaudii . . . . . . . . . . 27

Tringa alba . . . . . . . . . . . . 24

Tringa alpina . . . . . . . . . . . . 24

Tringa flavipes ............. . . 25

Tringa hiaticula . . . . . . . . . 24

Tringa himantopus . . . . . . . . . . . 24

Tringa lobata ... . . . . . . . . 25

Tringa longicauda . . . . . . . . . 24

Tringa macularia . . . . . . . . 24

Tringa melanoleuca . . . . . . . . 25

Tringa minutilla . . . . . . . . . 25

Tringa pusilla . . . . . . . . . 25

Tringa squatarola . . . . . . . . . . . . 24

Turdus cafer . . . . . . . . . . . . 28

Turdusfuscescens . . . . . . . . . . . 28

Turdus migratorius . . . . . . . . . 28

Turdus mustelinus . . . . . . . . . . . 28

Turdus trichas . . . . . . . . . . . . 29

Turdus ustulatus . . . . . . . . . . . . 28

Tympanuchus cupido . . . . . . . 23

Tympanuchus phasianellus . . . . . . 23

TYRANNIDAE . . . . . . . . . . 27

Tyrannus tyrannus . . . . . . . . . 27

Tyrannus verticalis . . . . . . . . . . . 27

Uria aalge . . . . . . . . . 26 26

Uria lomvia . . . . . . . . . . 26

Vermivora ruficapilla . . . . . . . . . . 29

Wild swans . . . . . . . . . . 20 20

Wilsonia canadensis . . . . . . . . . . . 29

Xanthocephalus xanthocephalus . . . . 30

Zonotrichia albicollis . . . . . . . 30

Zonotrichia leucophrys . . . . . . . . 30 



